\title{
uPAR-expressing melanoma exosomes promote angiogenesis by VE-Cadherin, EGFR and uPAR overexpression and rise of ERK1,2 signaling in endothelial cells
}

\author{
Alessio Biagioni ${ }^{1}$. Anna Laurenzana ${ }^{1}$ - Beatrice Menicacci ${ }^{1}$. Silvia Peppicelli ${ }^{1}$ - Elena Andreucci ${ }^{1}$. \\ Francesca Bianchini ${ }^{1}$ - Daniele Guasti ${ }^{2}$. Paolo Paoli ${ }^{1}$ - Simona Serrati ${ }^{3}$. Alessandra Mocali ${ }^{1} \cdot$ Lido Calorini $^{1}$. \\ Mario Del Rosso ${ }^{1} \cdot$ Gabriella Fibbi $^{1}$ - Anastasia Chillà ${ }^{1} \cdot$ Francesca Margheri $^{1}$ (D)
}

Received: 9 April 2020 / Revised: 2 November 2020 / Accepted: 6 November 2020 / Published online: 25 November 2020

(c) The Author(s) 2020

\begin{abstract}
Exosomes (Exos) have been reported to promote pre-metastatic niche formation, proliferation, angiogenesis and metastasis. We have investigated the role of UPAR in melanoma cell lines-derived Exos and their pro-angiogenic effects on human microvascular endothelial cells (HMVECs) and endothelial colony-forming cells (ECFCs). Melanoma Exos were isolated from conditioned media of A375 and M6 cells by differential centrifugation and filtration. Tunable Resistive Pulse Sensing (TRPS) and Nanoparticle tracking analysis were performed to analyze dimension and concentration of Exos. The CRISPR-Cas 9 technology was exploited to obtain a robust UPAR knockout. UPAR is expressed in melanoma Exos that are internalized by HMVECs and ECFCs, enhancing VE-Cadherin, EGFR and UPAR expression in endothelial cells that undergo a complete angiogenic program, including proliferation, migration and tube formation. uPAR loss reduced the pro-angiogenic effects of melanoma Exos in vitro and in vivo by inhibition of VE-Cadherin, EGFR and uPAR expression and of ERK1,2 signaling in endothelial cells. A similar effect was obtained with a peptide that inhibits uPAR-EGFR interaction and with the EGFR inhibitor Gefitinib, which also inhibited melanoma Exos-dependent EGFR phosphorylation. This study suggests that uPAR is required for the pro-angiogenic activity of melanoma Exos. We propose the identification of uPAR-expressing Exos as a potentially useful biomarker for assessing pro-angiogenic propensity and eventually monitoring the response to treatment in metastatic melanoma patients.
\end{abstract}

Keywords uPA/uPAR system $\cdot$ Exosomes $\cdot$ Melanoma cells $\cdot$ Angiogenesis $\cdot$ Endothelial cells

Abbreviations
EVs $\quad$ Extracellular vesicles
MVs $\quad$ Microvesicles

Alessio Biagioni and Anna Laurenzana contributed equally to the manuscript.

Electronic supplementary material The online version of this article (https://doi.org/10.1007/s00018-020-03707-4) contains supplementary material, which is available to authorized users.

Anastasia Chillà

anastasia.chilla@unifi.it

Francesca Margheri

francesca.margheri@unifi.it

1 Department of Experimental and Clinical Biomedical Sciences, Section of Experimental Pathology and Oncology, University of Florence, Viale G.B. Morgagni, 50,

50134 Florence, Italy

$\begin{array}{ll}\text { Exos } & \text { Exosomes } \\ \text { MVB } & \text { Multivesicular bodies } \\ \text { uPA } & \text { Urokinase plasminogen activator } \\ \text { uPAR } & \text { Urokinase plasminogen activator receptor } \\ \text { PAI-1 } & \text { Plasminogen activator inhibitor 1 } \\ \text { EGFR } & \text { Epidermal growth factor receptor } \\ \text { suPAR } & \text { Soluble plasminogen activator receptor } \\ \text { ECFCs } & \text { Endothelial colony-forming cells } \\ \text { HMVEC } & \text { Human microvascular endothelial cells }\end{array}$

2 Department of Experimental and Clinical Medicine, University of Florence, Viale Pieraccini 6, 50139 Florence, Italy

3 Nanotecnology Laboratory, National Cancer Research Centre, IRCCS “Giovanni Paolo II”, Viale Orazio Flacco, 65, 70124 Bari, Italy 


$\begin{array}{ll}\text { CRISPR } & \begin{array}{l}\text { Clustered regularly interspaced short palindro- } \\ \text { mic repeats }\end{array} \\ \text { TRPS } & \text { Tunable resistive pulse sensing } \\ \text { LAMP-1 } & \text { Lysosomal-associated membrane protein 1 } \\ \text { CM } & \text { Culture media } \\ \text { siRNA } & \text { Small interfering RNA } \\ \text { VE-Cad } & \text { Vascular endothelial cadherin } \\ \text { ECM } & \text { Extracellular matrix } \\ \text { MMP-12 } & \text { Matrix metallo-proteinase-12 }\end{array}$

\section{Introduction}

Melanoma is the deadliest skin cancer, with stage IV melanoma patients having a 5-year survival rate of less than $15 \%$ [1]. Despite significant advances in melanoma therapy, invasive melanoma incidence continues to rise worldwide faster than any other cancer [2] and it is responsible for approximately $70 \%$ of skin cancer-associated mortality [3]. The major cause of melanoma mortality is metastasis to distant organs, in lungs and brain [4]. In spite of many advances in understanding the cellular and molecular interactions within the tumor microenvironment, the underlying mechanism contributing to the generation of the pre-metastatic niche at distal sites remains elusive. Emerging evidence suggests that cancer-derived extracellular vesicles (EVs) play a major role not only in conditioning the tumor microenvironment but also in preparing the "soil" of the pre-metastatic niche for metastasis. [5, 6]. There are several types of EVs: microvesicles (MVs), apoptotic bodies and exosomes (Exos). Exos are nano-sized $(40-150 \mathrm{~nm})$ membrane-bound vesicles that originate from the late endosomal trafficking, are gathered intracellularly into multivesicular bodies (MVBs) and released by fusion with the plasma membrane [7]. They are critical mediators of intercellular communication between tumor and stromal cells via their biologically active payload, including proteins, lipids and metabolites, RNA and DNA $[8,9]$. Exos are reported to promote proliferation, invasion, and chemoresistance of cancer cells, to stimulate reprogramming of stromal cells to Cancer-Associated Fibroblasts (CAFs) and to promote angiogenesis which is critical for tumor cells release in the circulation and their spread to distant sites [10-13].

The uPA/uPAR system components (urokinase-type plasminogen activator, uPA; uPA receptor, uPAR; UPA inhibitor type-1, PAI-1) are actually considered important prognostic and predictive markers of malignancy [14]. Several malignant tumors show a positive correlation between UPAR levels, a more aggressive phenotype and a poor prognosis [15]. We have previously shown that UPAR is strongly up-regulated in A375 and in metastasis-prone M6 melanoma cells with respect to normal melanocytes [16]. uPAR overexpression in melanoma cells controls an invasive and glycolytic phenotype depending on alpha 5-beta1 integrin -mediated uPAR connection with EGFR [17]. We have also demonstrated that uPAR acquires a primary importance in vemurafenib resistance in tumors harboring the BRAF V600E mutation: high levels of UPAR and EGFR are associated with a lower sensitivity to vemurafenib [18]. At the same time, also the soluble urokinase-type plasminogen activator receptor (suPAR) has been proposed as a biomarker of tumor progression in several malignant tumors such as non-small-cell lung cancer [19], advanced breast cancer [20], colorectal cancer [21], ovarian cancer [22] and prostate cancer [23] and have been correlated with a poor prognosis.

Based on the above, in the current study, we aimed at addressing the role of uPAR in melanoma-derived Exos. We started to investigate the expression of UPAR both in ectosomes and Exos and the pro-angiogenic effects of melanoma Exos on human endothelial colony-forming cells (ECFCs) and on human microvascular endothelial cells (HMVECs). We have observed a quantitative increase of VE-Cadherin, EGFR and uPAR, along with a rise of EGFR phosphorylation and ERK1,2 signaling. We have further assessed the effects of CRISPR-Cas 9-mediated uPAR knockdown and of its rescue on the pro-angiogenic activity of melanoma-derived Exos in vitro and in vivo.

\section{Materials and methods}

\section{Cell lines and culture conditions}

The human melanoma cell line A375 (MITF wild type, BRAF V600E, NRAS wild type) was obtained from American Type Culture Collection (Manassas, VA) and was grown in Dulbecco's modified Eagle'smedium (DMEM, Euroclone, Milano, Italy) containing $2 \mathrm{mM}$ glutamine, $100 \mathrm{UI} / \mathrm{ml}$ penicillin, $100 \mu \mathrm{g} / \mathrm{ml}$ streptomycin and $10 \%$ FBS (Euroclone, Milano, Italy). A375-M6 melanoma cells (M6) were isolated from lung metastasis of SCID bg/bg mice i.v. injected with A375 cells and grown in the same conditions of A375. A375 and M6 were independently validated by STR profiling by the DNA diagnostic centre BMR Genomics (Padova, Italy). Cells were amplified, stocked, thawed and were kept in culture for a maximum of 4 months. ECFCs were isolated from $>50 \mathrm{ml}$ human umbilical cord blood (UCB) of healthy newborns as described previously [24], were selected as $\mathrm{CD}^{4} 5^{-}$, $\mathrm{CD} 34^{+}, \mathrm{CD} 31^{+}, \mathrm{CD}_{105^{+}}, \mathrm{ULEX}^{+}, \mathrm{vWF}^{+}, \mathrm{KDR}^{+}$cells [24] and were grown in EGM-2 culture medium (Lonza), supplemented with 10\% FBS. Human Microvascular Endothelial Cells (HMVECs) were purchased from Lonza and were grown in the same conditions of ECFCs. 


\section{Exosomes isolation}

Exos isolation protocol was modified from the previously published methods [25, 26]. See details in Supplemental Methods.

\section{TRPS (Tunable Resistive Pulse Sensing) analysis}

The size distribution and concentration of Exos were measured by TRPS analysis using a qNano platform with an NP100-rated nanopore (Izon Science, UK). Exosomal samples were diluted 1000-fold with PBS and measured three times. Data processing and analysis were carried out on the Izon Control Suite software (Izon Science, UK).

\section{Nanoparticle tracking analysis}

Nanoparticle tracking analysis was performed on a NanoSight NS300 (Malvern Panalytical, Westborough, MA, USA) equipped with a $488 \mathrm{~nm}$ excitation laser and an automated syringe sampler. NanoSight technology calculates size based on the relationship between Brownian motion and hydrodynamic diameter through the Stokes-Einstein equation. Exosomal samples were diluted 1:500 in PBS and loaded into $1 \mathrm{ml}$ syringes. CSV files generated by NTA by software v3.2 were used for computational analysis.

\section{Transmission electron microscopy (TEM)}

Negative staining technique was employed to visualize the Exos. An enriched exosomal suspension in filtered DPBS (Dulbecco's phosphate-buffered saline) was dispensed on carbon-coated electron microscopy grids on parafilm and left to absorb for $10 \mathrm{~min}$ at room temperature then transferred to a drop of Uranyless ${ }^{\circledR}$ solution for $1 \mathrm{~min}$ and left to air dry. Excess stain was blotted away. Imaging was performed using a JEOL 100CX II transmission electron microscope (TEM) at $100 \mathrm{kV}$.

\section{PKH67 labeling of Exos and exosomal uptake into recipient cells}

Melanoma-derived Exos were collected from $80 \mathrm{ml}$ of culture medium as described above. Exos were labeled using PKH67 Fluorescent Cell Linker kit (Sigma-Aldrich, St. Louis, MO) according to the manufacturer's instructions. Exos uptake was measured on a FACSCAN LSRII (Becton-Dickinson, excitation $=490 \mathrm{~nm}$, emission $=502 \mathrm{~nm}$ ) as previously described [27]. See details in Supplemental Methods.

\section{Immunofluorescence confocal microscopy}

Immunofluorescence was performed as previously described $[24,28]$. See details in Supplemental Methods.

\section{Cell proliferation assay}

$12 \times 10^{4}$ HMVECs and ECFCs/well were seeded in six-well plates. The day after plating, the standard culture medium was substituted with EBM plus 2\% FBS in the presence or absence of A375- and M6-derived Exos $(20 \mu \mathrm{g} / \mathrm{ml})$ (indicated as A375-Exos and M6-Exos, respectively) at final concentration of $20 \mu \mathrm{g} / \mathrm{ml}$. Cell proliferation was evaluated by cell counting at $24 \mathrm{~h}, 48 \mathrm{~h}, 72 \mathrm{~h}$ and $96 \mathrm{~h}$.

\section{Wound healing assay}

$12 \times 10^{4}$ HMVECs and ECFCs/well were seeded in sixwell plates and grown to confluency. The standard culture medium was then substituted with EBM plus 2\% FBS in the presence or absence of A375-Exos and M6-Exos at final concentration of $20 \mu \mathrm{g} / \mathrm{ml}$ and a wound was produced in each well with a $20-\mu l$ micropipette tip. Microphotographs of the wound were taken at time $0,6 \mathrm{~h}$ and $24 \mathrm{~h}$. Images were analyzed with the Image J MRI Wound healing software and reported as the percentage of the healing compared to initial wound area.

\section{Invasion assays in Boyden chambers}

Spontaneous invasion experiments were performed in Boyden chambers as previously described [24], with wells separated by $8-\mu \mathrm{m}$-pore size polycarbonate filters coated with Matrigel (50 $\mu \mathrm{g} /$ filter). See details in Supplemental Methods.

\section{Capillary morphogenesis}

In vitro capillary morphogenesis was performed as described [24], in tissue culture wells coated with Matrigel (BD Biosciences). ECFCs and HMVECs were resuspended $\left(18 \times 10^{3}\right.$ /well in 96-well plates) in EBM 2\% FBS in the presence or absence of A375-Exos and M6-Exos $(20 \mu \mathrm{g} / \mathrm{ml})$ and incubated for $6 \mathrm{~h}$ at $37{ }^{\circ} \mathrm{C}, 5 \% \mathrm{CO}_{2}$. Results were quantified at the end of experiment with Angiogenesis Analyzer tool of Image $\mathbf{J}$ software, measuring the number of junctions, branches, tubules, total length and total tubule length. Six to nine photographic fields from three plates were analyzed for each point. 


\section{Western Blot analyses}

Ectosome, Exos and cell aliquots of A375 and M6, as well as cell aliquots of control and Exos-treated ECFC and HMVEC cultures were processed for Western Blotting analyses. See details in Supplemental Methods.

\section{Cell treatment with M25 integrin antagonist peptide and Gefitinib}

Inhibition of uPAR-integrin interaction was obtained with the M25 peptide as previously described $[17,18]$, while EGFR phosphorylation was inhibited with Gefitinib, a specific inhibitor of the EGFR tyrosine kinase. See details in Supplemental Methods.

\section{siRNA for uPAR gene knockdown}

Targeting and not-targeting siRNAs were obtained from Dharmacon (Carlo Erba Reagents, Milan, Italy). Specific silencing of uPAR gene was performed, as previously described [17, 18]. See details in Supplemental Methods.

\section{Double Nickase Cas9 PLAUR gene knockout}

A complete PLAUR gene knockout was obtained, as previously described [29], by transfection of A375 and A375-M6 with two CRISPR/Cas9 D10A plasmids, each one bearing a specific sgRNA designed by the manufacturer to generate a double strand break in UPAR exon 3. For uPAR expression rescue experiments, cells were stably transfected using an Okayama-Berg vector containing uPAR cDNA and selected with $\mathrm{G} 418$ as resistance marker $(0.5 \mathrm{mg} / \mathrm{ml})$ as previously reported $[29,30]$.

\section{In vivo Matrigel plug assay}

All procedures involving animals were performed in accordance with the ethical standards and according to the Declaration of Helsinki and to national guidelines approved by the ethical committee of Animal Welfare Office of Italian Health Ministry and conformed to the legal mandates and Italian guidelines for the care and maintenance of laboratory animals. Five hundred $\mu$ of Matrigel (BD Biosciences) mixed with $(20 \mu \mathrm{g} / \mathrm{ml})$ Exos from wild type, uPAR ko- and uPAR rescued M6 was injected subcutaneously in the ventral region of nude mice ( 12 mice, 4 animals for each condition) (Charles River). After 7 days, the Matrigel was excised and then fixed with formalin overnight, embedded in paraffin,
A
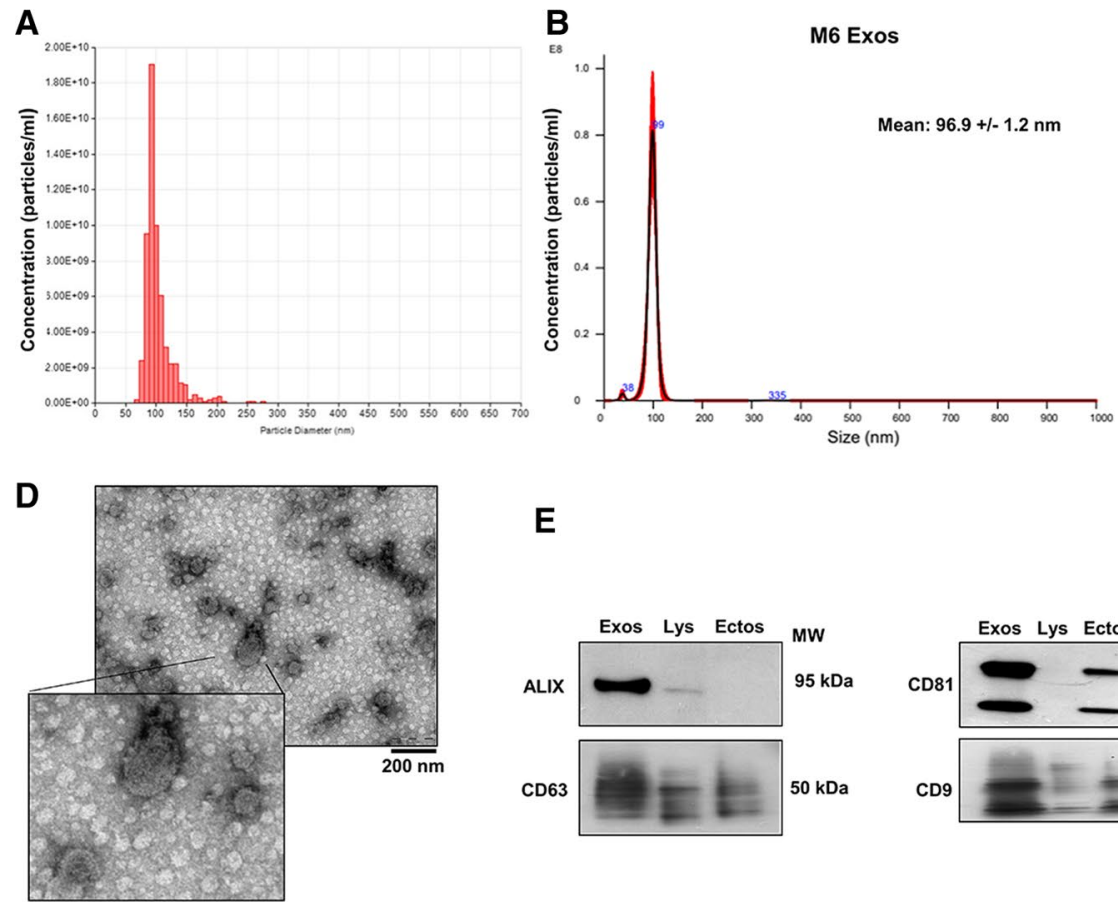

E

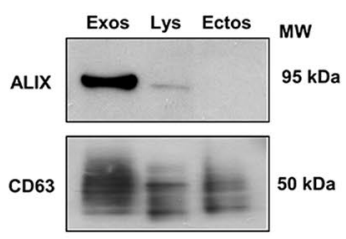

C

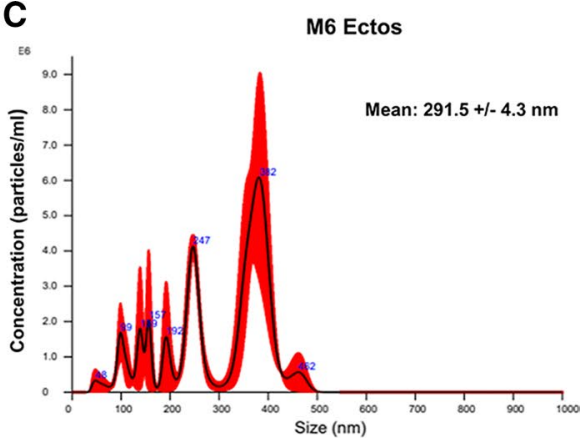

F

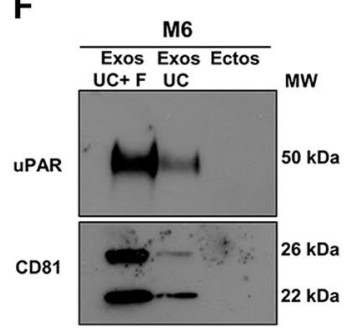

Fig. 1 Characterization of M6-derived exosomes and ectosomes. a Particles size distribution and concentration by TRPS of M6-Exos. b, c Nanoparticles Tracking Analysis of exosomes (Exos) and ectosomes (Ectos), respectively, by Nanosight technology. d Morphology and dimension of M6-Exos under a transmission electron microscopy.
Scale bar: $200 \mathrm{~nm}$. e Western blotting analysis of exosomal marker proteins (including ALIX, CD63, CD81, CD9). f Western Blotting of uPAR in M6-Exos and ectosomes. CD81 was used as loading control. $M W$ molecular weight; Exos exosomes; Lys total lysate; Ectos Ectosomes; $U C$ ultracentrifugation; $F$ filtration 


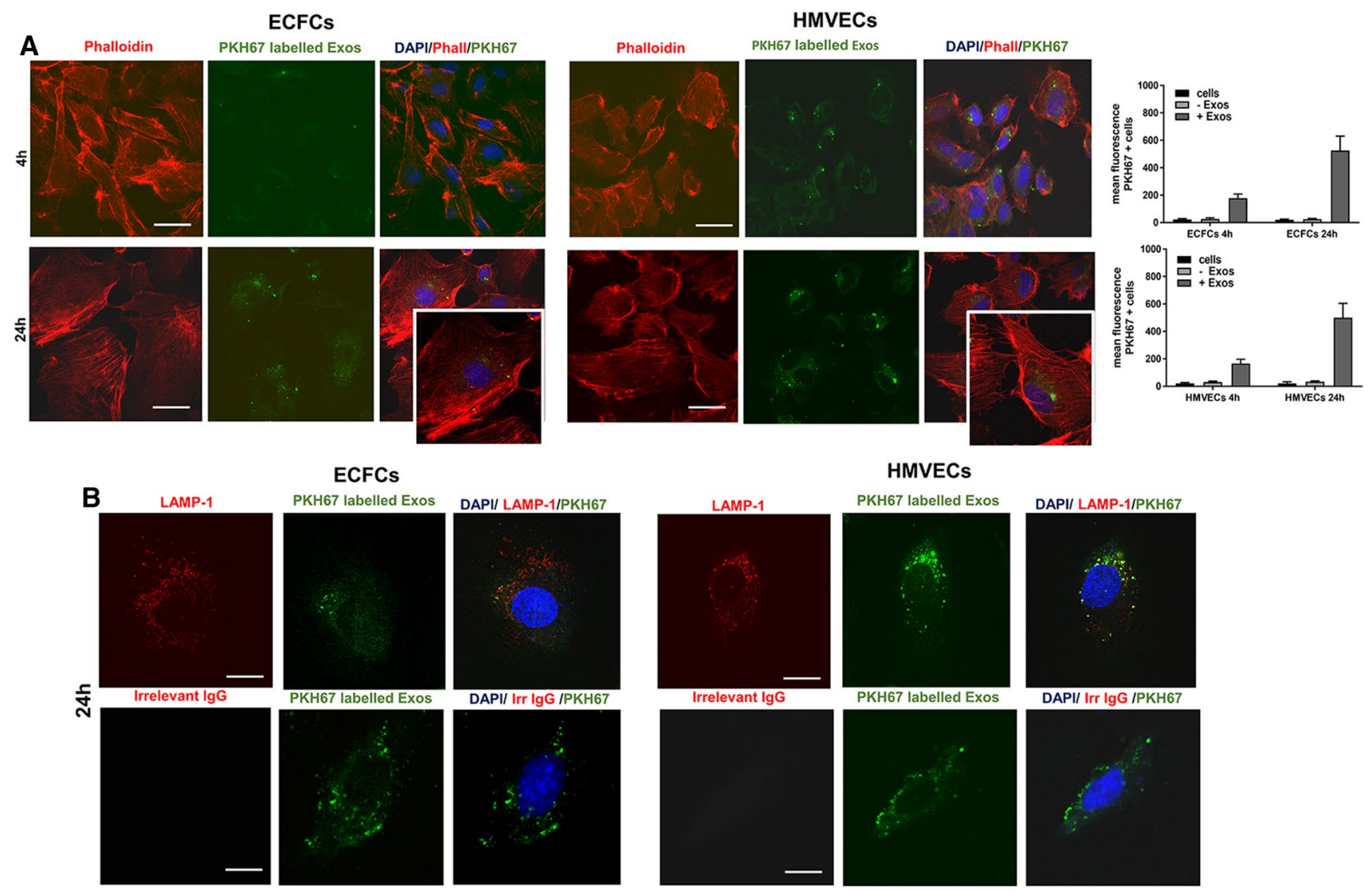

Fig. 2 Internalization of M6-Exos into ECFCs and HMVECs. a Localization by confocal microscopy analysis of PHK67-labeled M6-Exos in ECFCs and HMVECs stained with TRITC-labeled phalloidin. The green-labeled Exos were visible in the perinuclear region of HMVECs and ECFCs. Scale bar: $20 \mu \mathrm{m}$. Histograms on

and sectioned to obtain slides. The plugs were stained with hematoxylin and eosin (H\&E) and visualized using the Zeiss inverted microscope (Zeiss, Germany). In vivo neovascularization was quantified by blood vessel density using Image J software.

\section{Statistical analysis}

Statistical analyses of the data were performed using oneway ANOVA, and $p \leq 0.05$ was considered a statistically significant difference; while $p \leq 0.01$, a very statistically significant difference.

\section{Results}

\section{Isolation and characterization of melanoma-derived Exos}

Melanoma-derived Exos were isolated from culture media (CM) of A375 and M6, the metastatic clone of A375, after the right represent the PHK67 mean fluorescence intensity of ECFCs and HMVECs after 4-h and 24-h incubation with stained exosomes (+(Exos) or an exosome-negative control (-Exos). b Confocal microscopy analysis of PHK67-labeled M6-Exos and LAMP-1, a lysosome marker. Scale bar: $10 \mu \mathrm{m}$

$48 \mathrm{~h}$ incubation in serum-free media by differential centrifugation and filtration. In order to identify the purified Exos, we characterized our population by TRPS and Nanoparticle tracking analysis. TRPS analysis (Fig. 1a) showed the distribution and concentration of M6-Exos. The size of M6-Exos was approximately ranging from 50 to $150 \mathrm{~nm}$, concordant with the previously reported exosomal size distribution [26]. Similar results were obtained with Nanoparticle tracking analysis performed by Nanosight (mean: $96.9 \pm 1.2 \mathrm{~nm}$ ) (Fig. 1b). Nanoparticle tracking analysis of ectosomes (Fig. 1c) showed several components with different dimension. The mean size of this distribution was $291.5 \pm 4.3 \mathrm{~nm}$ according to the previous observations [31]. TEM analysis revealed, as evident in Fig. 1d, that M6-Exos have a round-, cup-shaped morphology with a diameter ranging from 50 to $150 \mathrm{~nm}$, consistent with the data of TRPS and Nanoparticle tracking. Western Blotting analyses indicate that M6-Exos were positive for the characteristic exosomal surface marker proteins (CD9, CD63, and CD81, members of tetraspanin family) and ALIX, a component of Multivesicular Body (MVB), expressed only in Exos [10], confirming 
the efficacy of purification methods (Fig. 1e). Similar results were obtained in A375-Exos (Supplementary Fig. S1, panels $\mathrm{A}, \mathrm{B}, \mathrm{C}$ and D).

\section{UPAR expression in melanoma-derived Exos}

UPAR is an important prognostic and predictive marker in melanoma progression $[16,17]$. We have previously shown that uPAR is strongly up-regulated in A375 and M6 melanoma cells with respect to human melanocytes [16]. To investigate the expression of UPAR in melanoma-derived EVs, we analyzed uPAR levels in both ectosomes (collected from $\mathrm{CM}$ after $12000 \times g$ centrifugation) and Exos (collected from $\mathrm{CM}$ after filtration and $100,000 \times g$ ultracentrifugation). Western Blotting analyses (Fig. 1f) show that uPAR is present only in Exos but not in ectosomes and, in particular, we observed an enrichment of uPAR in the smaller vesicles obtained by ultracentrifugation combined with filtration compared to those collected after ultracentrifugation only. CD81 was used as loading control. Similar results were obtained in A375 (Supplementary Fig. S1, panel E).
Fig. 4 Effects of M6-Exos and A375-Exos on tube formation and angiogenesis-related signaling in ECFCs and HMVECs. Capillary morphogenesis assays in the presence or absence of M6-Exos (a) and A375-Exos (b). Representative microphotographs $(\times 10)$ of capillary-like structures are shown. Quantification of capillary network by Angiogenesis Analyzer Image $\mathbf{J}$ tool. Histograms represent the mean number of master junctions, branches, tubules, total length, and total tubule length, respectively. Data are representative of measures obtained from at least nine fields. * Shows statistical significance $(p<0.05)$ compared to untreated cells. c Western Blotting analyses of KDR, VE-Cad, EGFR, uPAR, pERK, ERK1,2. GAPDH and tubulin were used as a loading control. Densitometric quantification of the immunoblots normalized to the relative internal control and expressed respect to untreated (- M6 and A375 exos) ECFCs and HMVECs is reported on the right. $M W$ molecular weight

\section{Internalization of A375- and M6-Exos by HMVECs and ECFCs}

After melanoma Exos characterization, we assessed the effects of A375- and M6-Exos on the angiogenic activities of endothelial cells in vitro. First, we determined whether melanoma Exos could be internalized into endothelial cells. To this purpose, A375- and M6-Exos were labeled by a

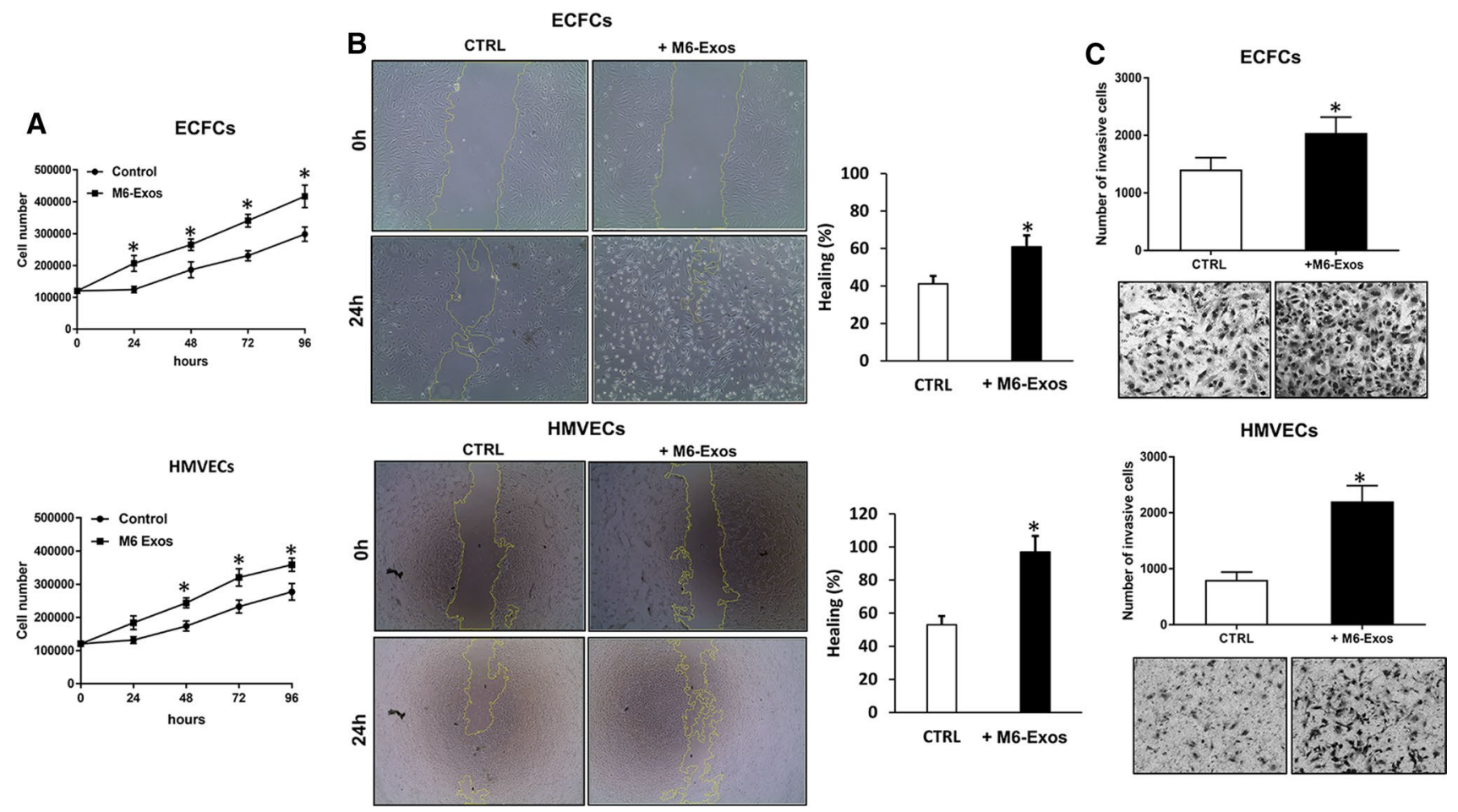

Fig. 3 Effects of M6-Exos on proliferation, migration and invasion of ECFCs and HMVECs. a Cell proliferation in the presence or absence of M6-Exos analyzed by cell counting at 24, 48, 72 and $96 \mathrm{~h}$. Results are reported as mean \pm SD of three different experiments. b Wound healing assays in the presence or absence of M6-Exos. Microphotographs $(\times 10)$ at 0 and $24 \mathrm{~h}$ from the wound are shown. Histograms represent the percentage of the healing (the mean of three different experiments $\pm S D$ is reported) measured after incubation with or without M6-Exos. * shows statistical significance $(p<0.05)$ compared to untreated cells. c Spontaneous invasion. $2.5 \times 10^{4}$ cells were suspended in the reference media in the presence or absence of M6-Exos and placed in the upper well. Fresh EBM was placed in the lower well. Data are reported as the number of migrated cells after incubation with M6-Exos suspended in EBM, compared to those migrated after incubation with fresh EBM alone. All histograms represent the mean of three different experiments \pm SD. Representative microphotographs $(\times 10)$ of migrated cells are shown under the respective histogram 

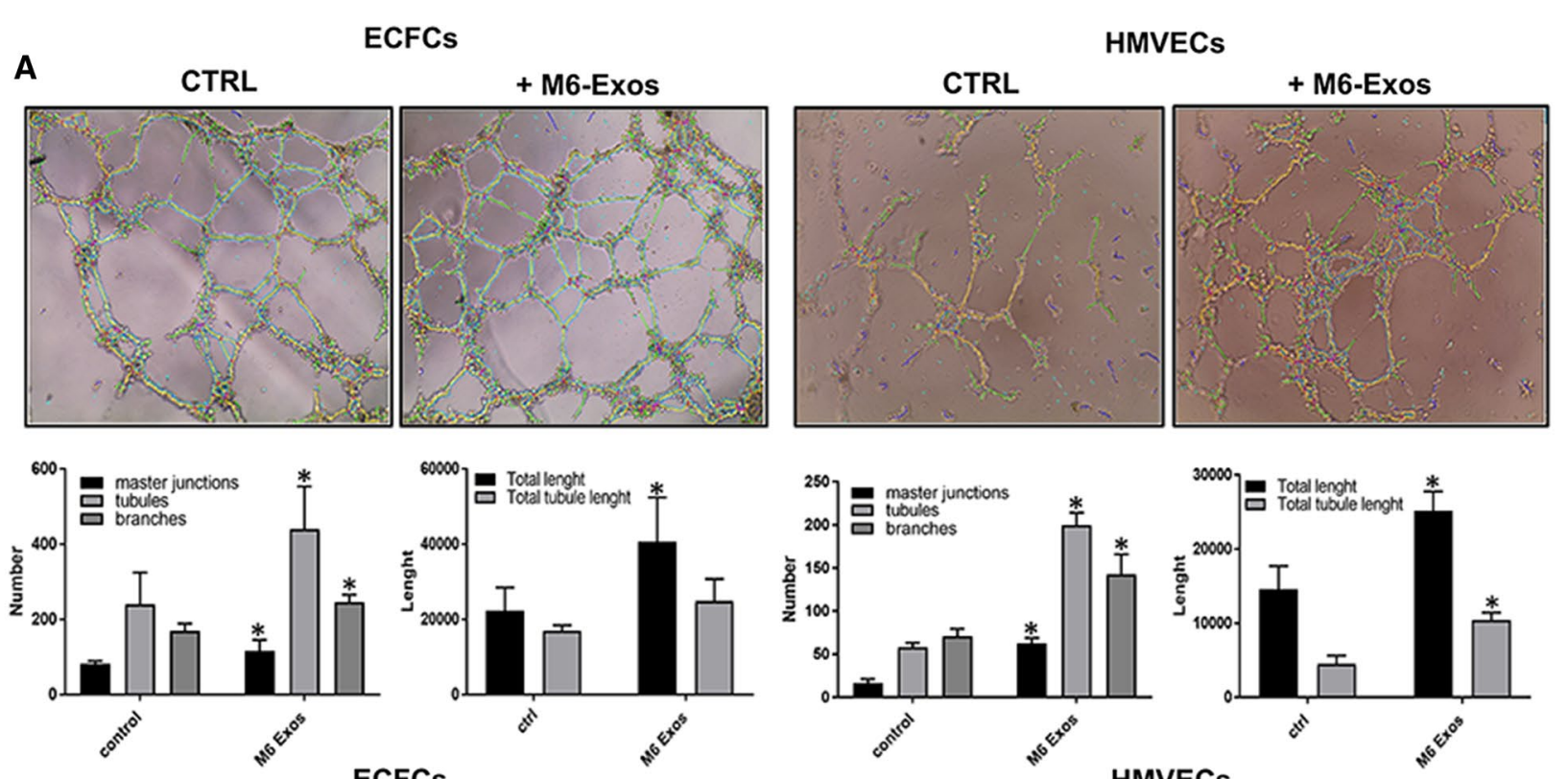

ECFCs
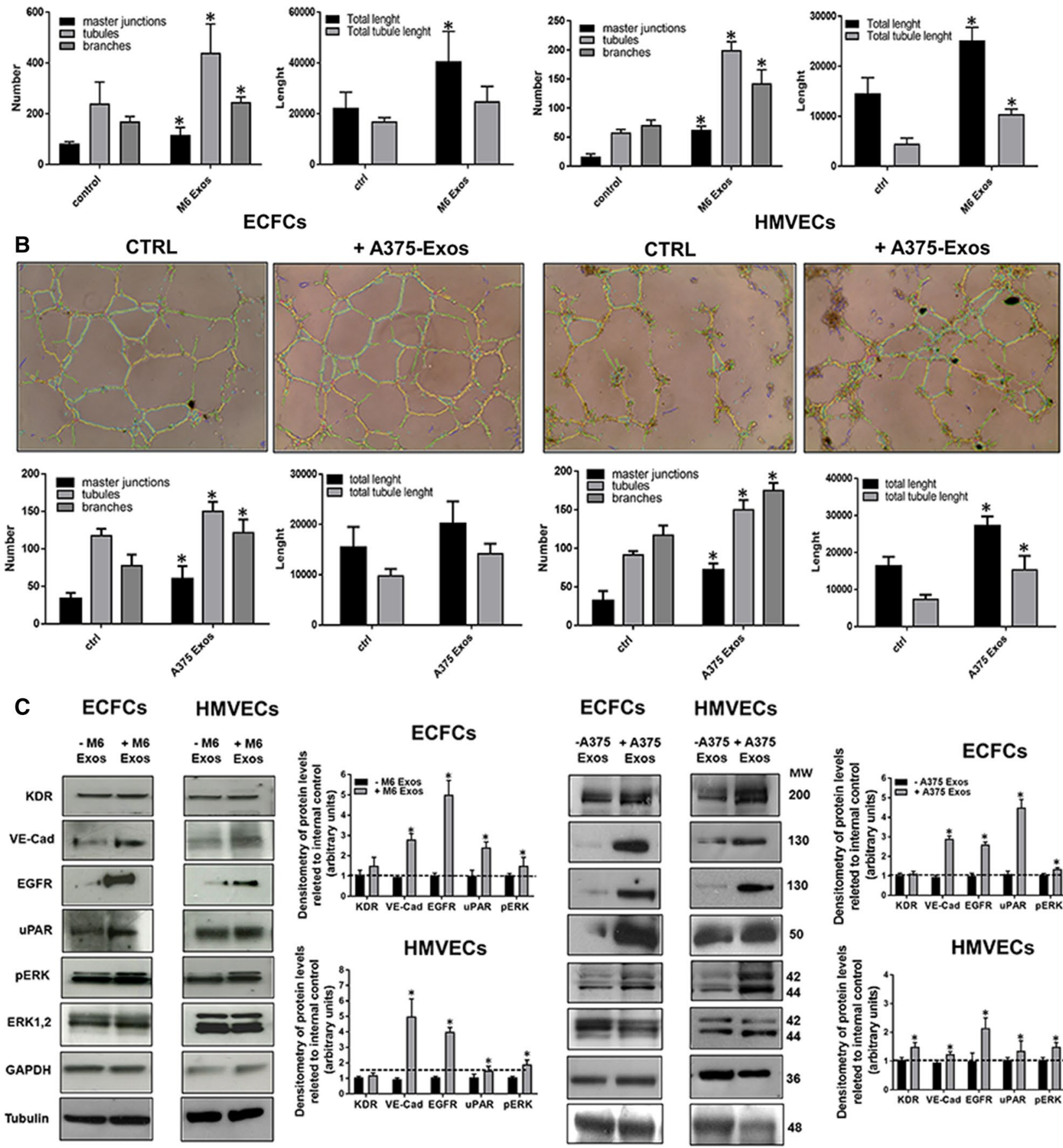

\section{ECFCs HMVECs}

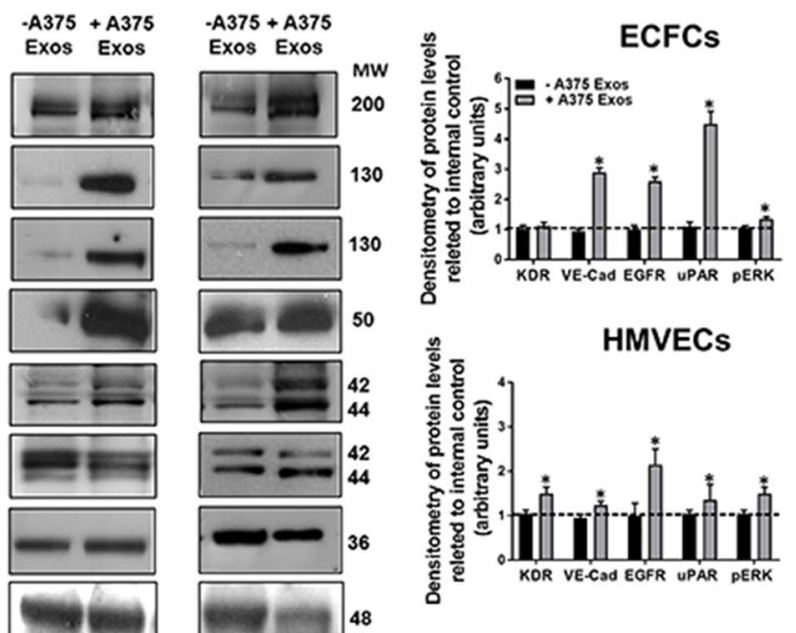


A

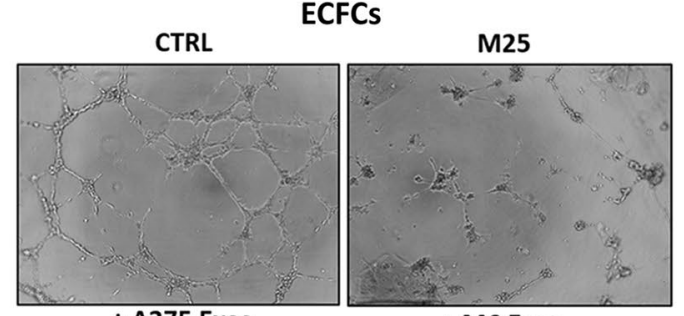

$+\mathrm{A} 375$ Exos

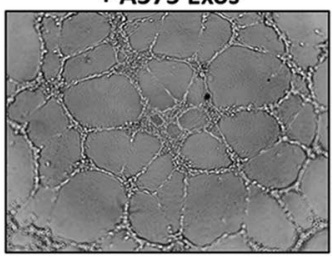

$+\mathrm{A} 375$ Exos + M25

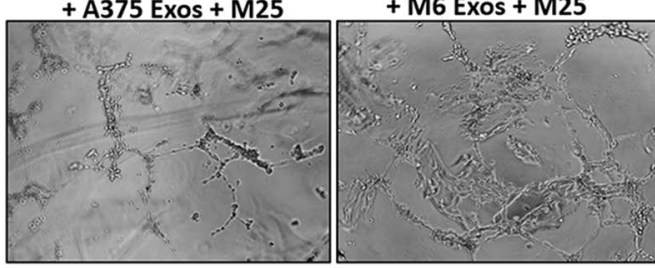

B
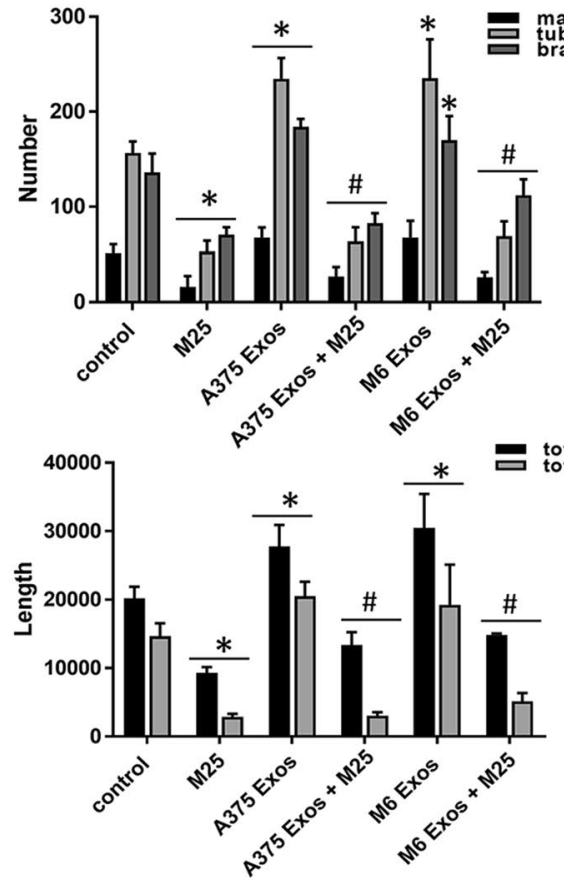

HMVECS
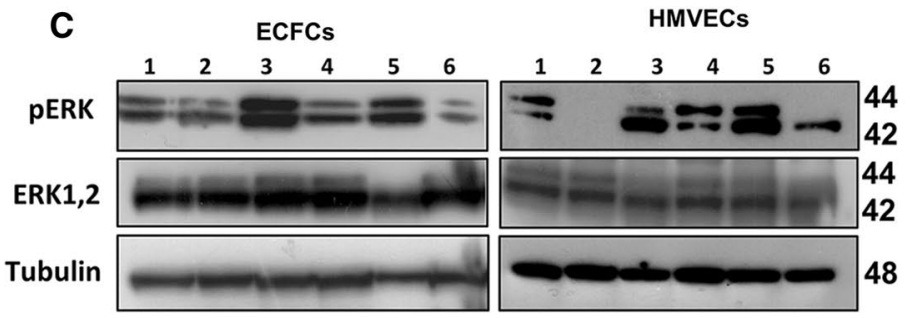

1= ctrl, 2=M25, 3= M6 Exos, 4= M6 Exos + M25, 5 = A375 Exos, 6 = A375 Exos + M25

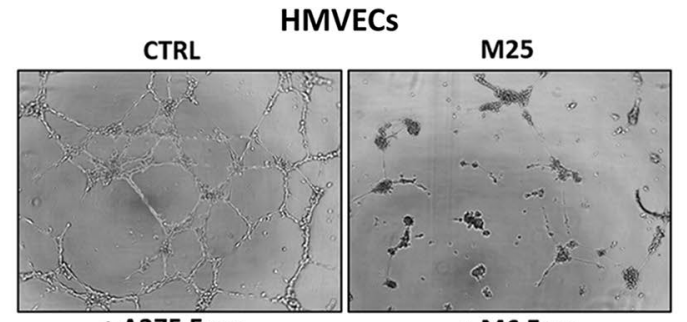

$+\mathrm{A} 375$ Exos

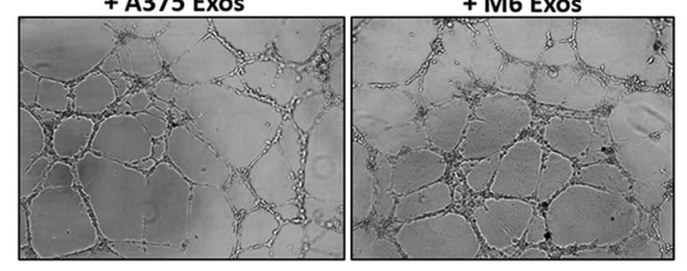

+ A375 Exos + M25

+ M6 Exos + M25
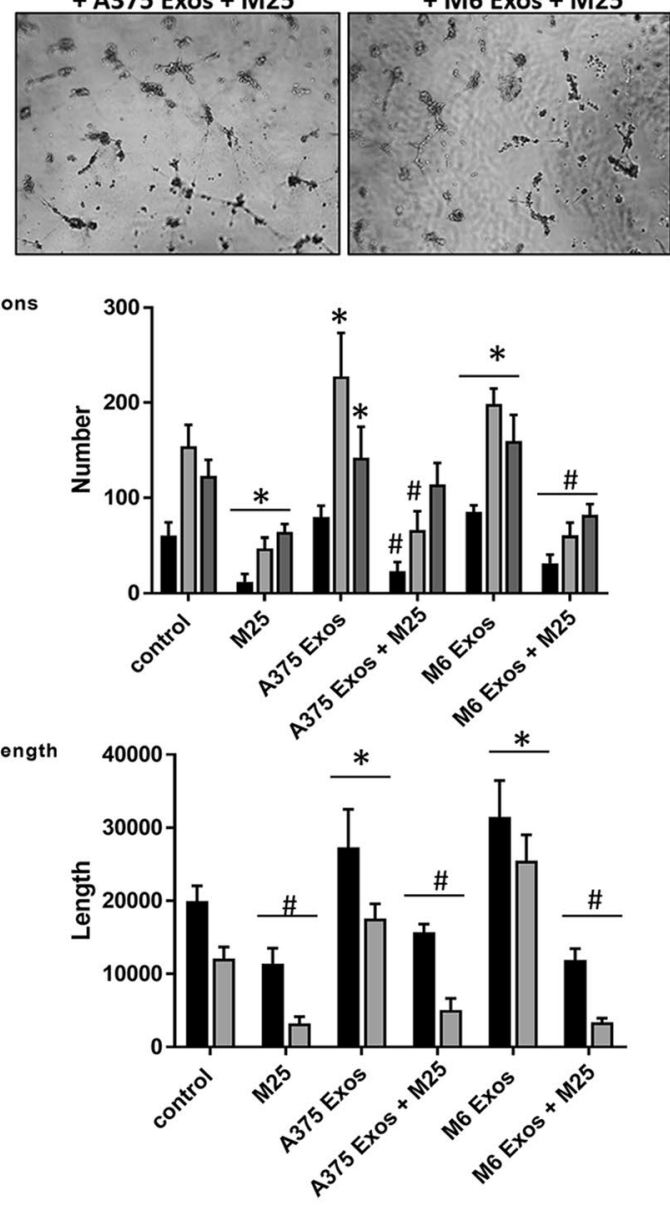

HMVECs
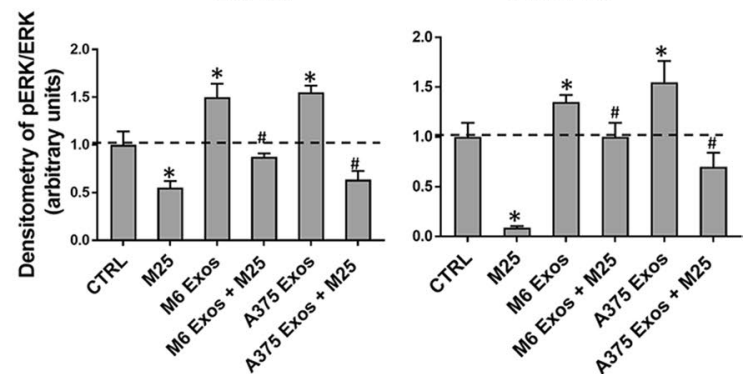
4Fig. 5 Activity of M25 peptide on the pro-angiogenic effects of melanoma- Exos. Capillary morphogenesis assays of M6-Exo and A375-Exo-treated ECFCs and HMVECs in the presence of absence of M25 peptide. a Representative microphotographs $(\times 10)$ of capillary-like structures are shown. b Quantification of capillary network by Angiogenesis Analyzer Image $\mathbf{J}$ tool. Histograms represent the mean number of master junctions, branches, tubules, total length, and total tubule length, respectively. Data are representative of measures obtained from at least nine fields. * Shows statistical significance $(p<0.05)$ compared to untreated cells. $\mathbf{c}$ Western Blotting analyses of p ERK and ERK1,2. Tubulin was used as a loading control. Densitometric quantification of the immunoblots normalized to the relative internal control and expressed respect to untreated (- M6 and A375 exos) ECFCs and HMVECs is reported on the right. $M W$ molecular weight

green fluorescent lipophilic dye (PHK67) and HMVECs and ECFCs were incubated with green Exos for 4 and $24 \mathrm{~h}$, then stained with phalloidin and analyzed by immunofluorescence for LAMP-1, a lysosome marker. After $24 \mathrm{~h}$, the labeled M6-Exos were evident in the perinuclear region of endothelial cells at the lysosomal compartment, indicating that M6-Exos were efficiently internalized into ECFCs and HMVECs. (Fig. 2a and b. To quantify the exosome uptake in endothelial cells, we performed flow cytometry analysis. The results (Fig. 2a, on the right) confirmed our previous microscopy observations and showed that the uptake of exosomes was time dependent. Indeed, the mean fluorescence intensity of PHK67 + endothelial cells results increased after 24-h incubation with M6-labeled exosomes. Similar results were obtained with A375-Exos (Supplementary Fig. S2).

\section{Pro-angiogenic effects of A375- and M6-Exos on endothelial cells}

To explore the functional role of melanoma Exos on angiogenesis, HMVECs and ECFCs were treated with A375- and M6-Exos for a series of in vitro angiogenesis-related assays: proliferation, migration and invasion. The effect of M6-Exos on the proliferation was analyzed by cell counting at 24,48 , 72 and $96 \mathrm{~h}$. M6-Exos significantly enhanced (about 50\% for ECFCs and $40 \%$ for HMVECs, respectively) the proliferation of HMVECs and ECFCs compared to untreated cells at any time of observation (Fig. 3a). A scratch wound healing assay was performed to assess the effect of M6-Exos on the migration of endothelial cells. The results of wound closure quantification (\% of healing) showed in Fig. 3b demonstrated that exosomal treatment remarkably increased the motility of endothelial cells. Similar results were obtained in Matrigel invasion assay in Boyden chambers (Fig. 3c). Indeed, Exostreated ECFCs and HMVECs showed an increase of invasion properties compared to untreated cells. Likewise, A375-Exo treatment enhanced ECFC and HMVEC proliferation and motility (migration and invasion) (Supplementary Fig. S3).
Lastly, to investigate the effect of A375- and M6-Exos on the angiogenic tubule formation, we performed capillary morphogenesis assays in Matrigel, in the presence or absence of A375- and M6-Exos. As shown in Fig. 4a and $b$, the number of master junctions, branches, tubules, total length e total tubule length were significantly increased in Exo-treated HMVECs and ECFCs and a better capillary network was observed compared to untreated cells. Therefore, taken together, our in vitro functional assays indicate that A375- and M6-Exos could activate a proangiogenic response in recipient endothelial cells. To investigate the mechanism through which A375- and M6-Exos could modulate endothelial cell function, we analyzed by Western Blotting the cell signaling transductions involved in angiogenesis. Figure $4 \mathrm{c}$ shows that Exos treatment induced a relevant increase of uPAR, EGFR and VE-Cadherin protein levels and a slight increment of KDR expression. These features were coupled with an increase of ERK1,2 (p42/44) phosphorylation in both ECFCs and HMVECs. Both morphological and signaling differences induced by A375- and M6-Exos were inhibited by the integrin antagonist peptide M25, that uncouples integrinmediated uPAR-EGFR interaction, as previously shown [18] (Fig. 5, panels a, b and c). To prove the direct role of EGFR in Exo-mediated angiogenesis, we performed capillary morphogenesis in the presence or absence of Gefitinib, an EGFR inhibitor which interrupts the signaling through EGFR in target cells. As shown in Fig. 6 panel a, Gefitinib reduced the pro-angiogenic effects of A375and M6-Exos in ECFCs and HMVECs. In parallel, in the presence of Gefitinib, we observed an inhibition of Exosmediated EGFR phosphorylation. (Fig. 6 panel b). Taken together, these data indicate that the UPAR interactome transferred by melanoma exos in target endothelial cells could play a prominent role in melanoma-associated tumor angiogenesis by control of EGFR signaling.

\section{UPAR is required for the pro-angiogenic activity of A375- and M6-Exos}

To establish a direct correlation between uPAR expression in melanoma Exos and their pro-angiogenic effects, we performed siRNA-mediated uPAR silencing in M6. After validation of uPAR down-regulation by Western Blotting (Supplementary Fig. S4A), we purified Exos from siCONTROL and siPLAUR M6 CM, respectively. Supplementary Fig.S4B shows a consistent inhibition of capillary-like structures in siPLAUR-Exos-treated compared to siCONTROL-Exos-treated ECFCs. To further validate the evidence that uPAR is critical for the Exos-mediated angiogenesis, we have exploited the CRISPR-CAS9 technology to obtain a robust irreversible uPAR gene knockout in A375 and M6 (Fig. 7a, left panel), as recently published [29]. In parallel, 


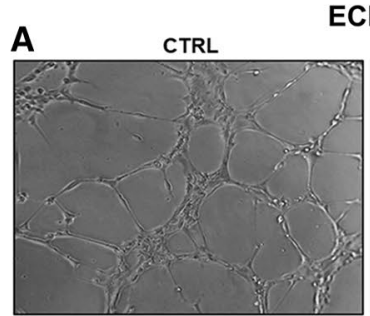

+ A375 Exos

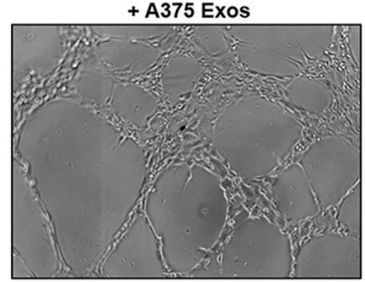

+ A375 Exos + Gefitinib
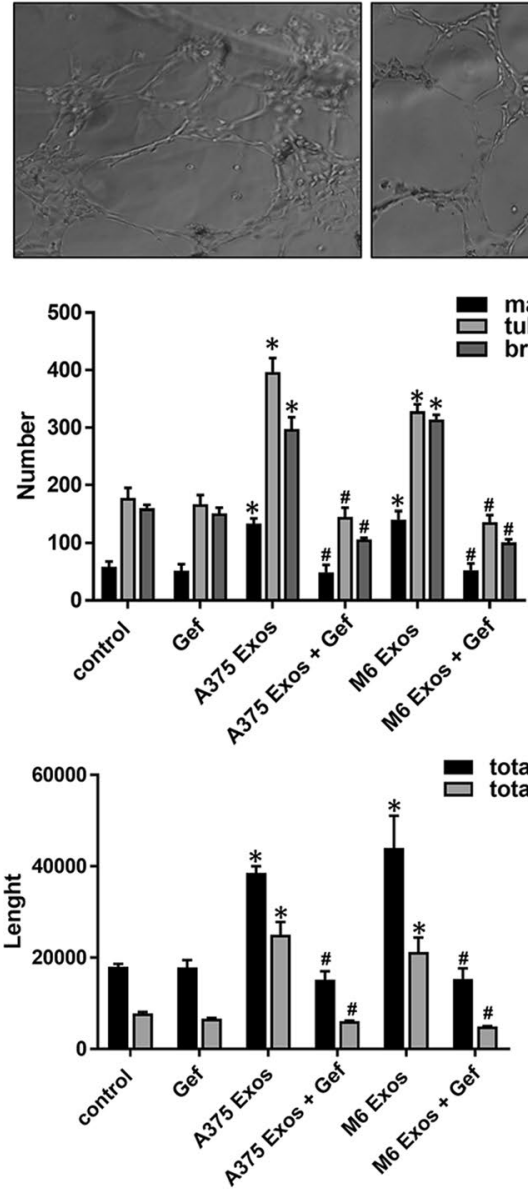

B
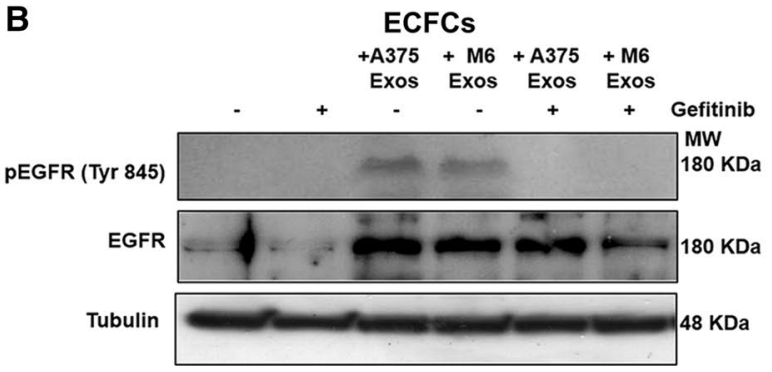

HMVECS

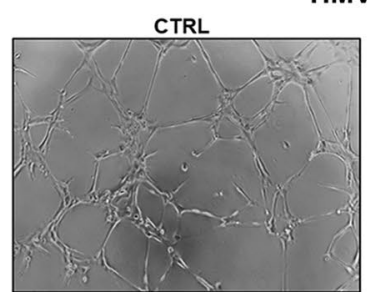

+ A375 Exos

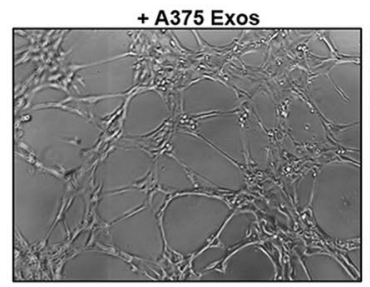

+A375 Exos + Gefitinib

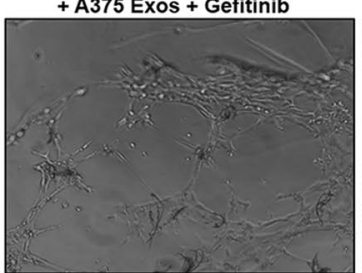

+ A375 Exos + Gefitinib
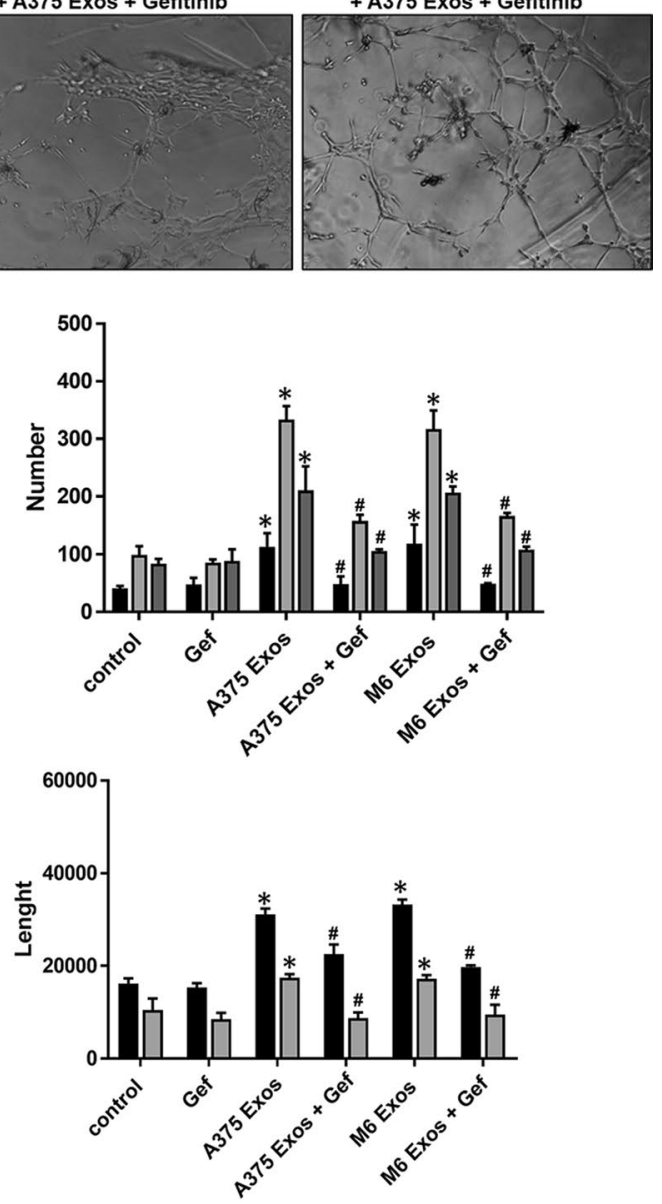

HMVECS

$+\mathrm{A} 375+\mathrm{M} 6+\mathrm{A} 375+\mathrm{M} 6$

Exos Exos Exos Exos

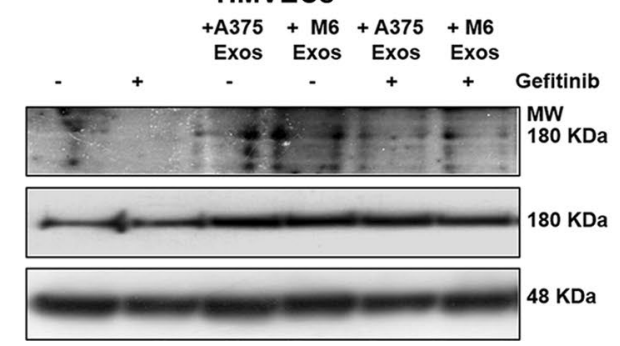


4Fig. 6 Anti-Exos pro-angiogenic activity of Gefitinib and inhibition of Exos-dependent EGFR phosphorylation. Capillary morphogenesis assays of M6-Exo and A375-Exo-treated ECFCs and HMVECs in the presence of absence of Gefitinib $10 \mu \mathrm{m}$. a Representative microphotographs $(\times 10)$ of capillary-like structures are shown. b Quantification of capillary network by Angiogenesis Analyzer Image $\mathrm{J}$ tool. Histograms represent the mean number of master junctions, branches, tubules, total length, and total tubule length, respectively. Data are representative of measures obtained from at least nine fields. * Shows statistical significance $(p<0.05)$ compared to untreated cells. $\mathbf{c}$ Western Blotting analyses of pEGFR and EGFR. Tubulin was used as a loading control. $M W$ molecular weight

to directly demonstrate the role of uPAR, we performed an experiment of expression rescue (Fig. 7a, right panel). Exos were collected from CM of uPAR ko and uPAR Rescue (uPAR+) A375 and M6 and capillary morphogenesis assays were performed. We observed a significant reduction of capillary network in the presence of uPAR ko-Exos both in HMVECs and in ECFCs that was restored in the presence of uPAR+-Exos (Fig. 7b). The levels of VE-Cad and pERK1,2 (Fig. 6c) were remarkably reduced in ko-Exostreated HMVECs and ECFCs compared to control and, on the other hand, increased in uPAR +-Exo-treated endothelial cells. Similar results were obtained in A375 (Supplementary Fig. S5). These results suggest that uPAR is critical for the pro-angiogenic effects of melanoma-derived Exos through a ERK1,2-mediated pathway.

\section{UPAR + melanoma Exos promote angiogenesis in vivo}

To validate our observations in vivo, we further assessed the angiogenic potential of Exos by examining the recruitment of endothelial cells and vasculature formation within subcutaneously implanted Matrigel plugs containing Exos derived from wild type, uPAR ko and uPAR Rescue (uPAR+) M6 and A375. The plugs containing uPAR+-Exos become more vascularized than implants with uPAR ko- and wild-type Exos (Fig. 8a). Histological examination by Hematoxylin and Eosin staining indicates that Matrigel plugs with uPAR+-Exos showed more micro-vessels compare to plugs containing uPAR ko and wild-type Exos. Indeed, the vascular density of Matrigel plugs (Fig. 8b) with uPAR+-Exos was significantly higher than those containing uPAR ko, that not contain blood vessels, and wild-type Exos. These data suggest that wild-type and uPAR+-Exos play an evident role for endothelial cells recruitment and vascular organization in vivo. So, uPAR expression is important for the angiogenic potential of A375- and M6-Exos both in vitro and in vivo.

\section{Discussion}

Accumulating evidence indicate that Exos are important in melanoma progression, supporting pro-tumoral processes including angiogenesis, immune regulation and modification of tumor microenvironment [11]. In addition, Exos may also vehiculate pro-angiogenic molecules that promote neoangiogenesis and ECM remodeling [32]. The formation of a new vascular network and remodelling of the extracellular space are crucial for the detachment of melanoma cells from the primary site, which represents the first steps of the metastatic cascade.

In the present study, we have investigated the role of uPAR in Exos derived from melanoma cells and their proangiogenic effects in HMVECs and ECFCs. First, we have isolated Exos from A375 and M6, derived from a lung metastasis of A375, by ultracentrifugation and filtration. Then, we have characterized the purified Exos in terms of dimensions, integrity and the typical protein markers expression. A375- and M6-Exos appeared as round-, cup-shaped EVs, ranging from 50 to $150 \mathrm{~nm}$, and expressing CD63, CD81, CD9 and Alix. These data are consistent with what has been reported for Exos from other melanoma cell lines [33]. In addition, we analyzed the expression of UPAR, an important prognostic and predictive marker of malignancy in melanoma cells $[14,16]$. Here, we demonstrated that uPAR is expressed in Exos but not in ectosomes. A possible explanation of this differential expression may be found in UPAR localization in specialized membrane microdomains, called lipid rafts. We have previously shown that the angiogenic properties of ECFCs depend on the integrity of caveolae and on the presence of full-length UPAR in such structures [24]. We have also demonstrated that UPAR binds preferentially to ganglioside GM1-enriched membranes, promoting invasion and capillary morphogenesis in ECFCs [34]. As reported by Thuma F. et al. [35], uPAR associates with palmitoylated claudin7, a major component of tight junctions, located in tetraspanin-enriched microdomains, similar to glycolipid-enriched microdomains (GEM). These specialized membrane regions, prone for internalization [36, 37], are recruited into early endosomes and these complexes are maintained and recovered in Exos [38-40]. In addition, Endo-Munoz L et al. [41] have observed that, in metastatic osteosarcoma, uPA was secreted in an active Exos-bound form, influencing metastatic behavior via locally secreted uPA and at distant sites via uPA-containing Exos. Therefore, it is likely that uPAR may reside in Exos rather than in ectosomes. After their characterization, we studied the ability of melanoma Exos to stimulate an angiogenic program in endothelial cells. We have shown that A375- and M6-Exos are internalized and are able to promote the angiogenic properties of endothelial cells stimulating in vitro proliferation, 
A

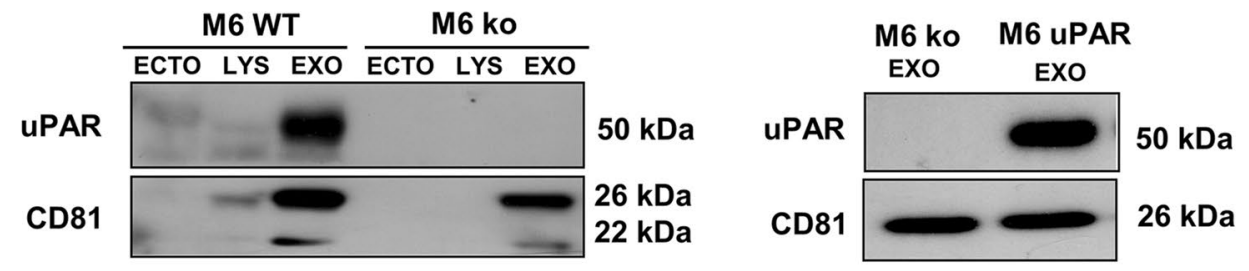

B

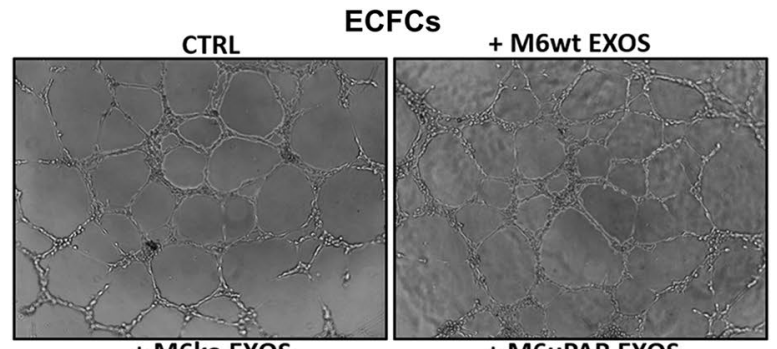

+ M6ko EXOS

+ M6uPAR EXOS
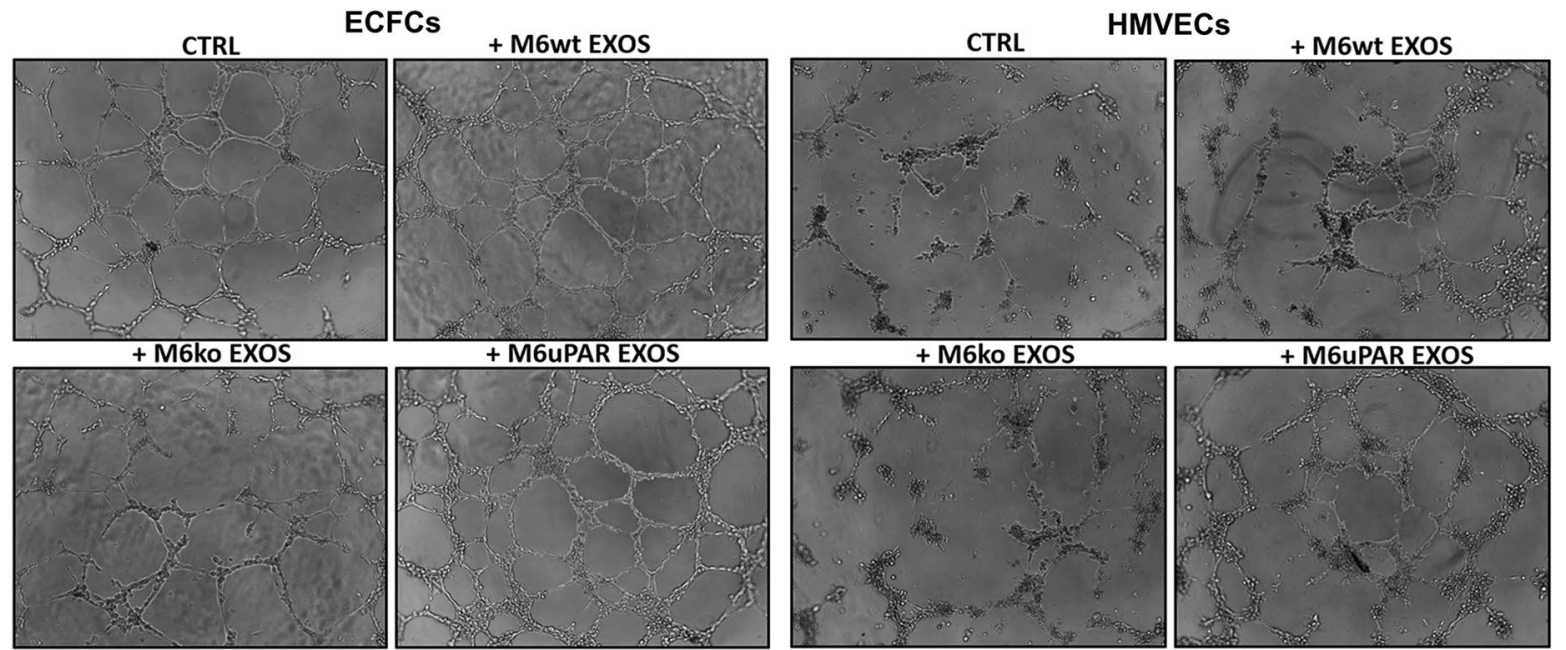

+ M6ko EXOS

+ M6uPAR EXOS
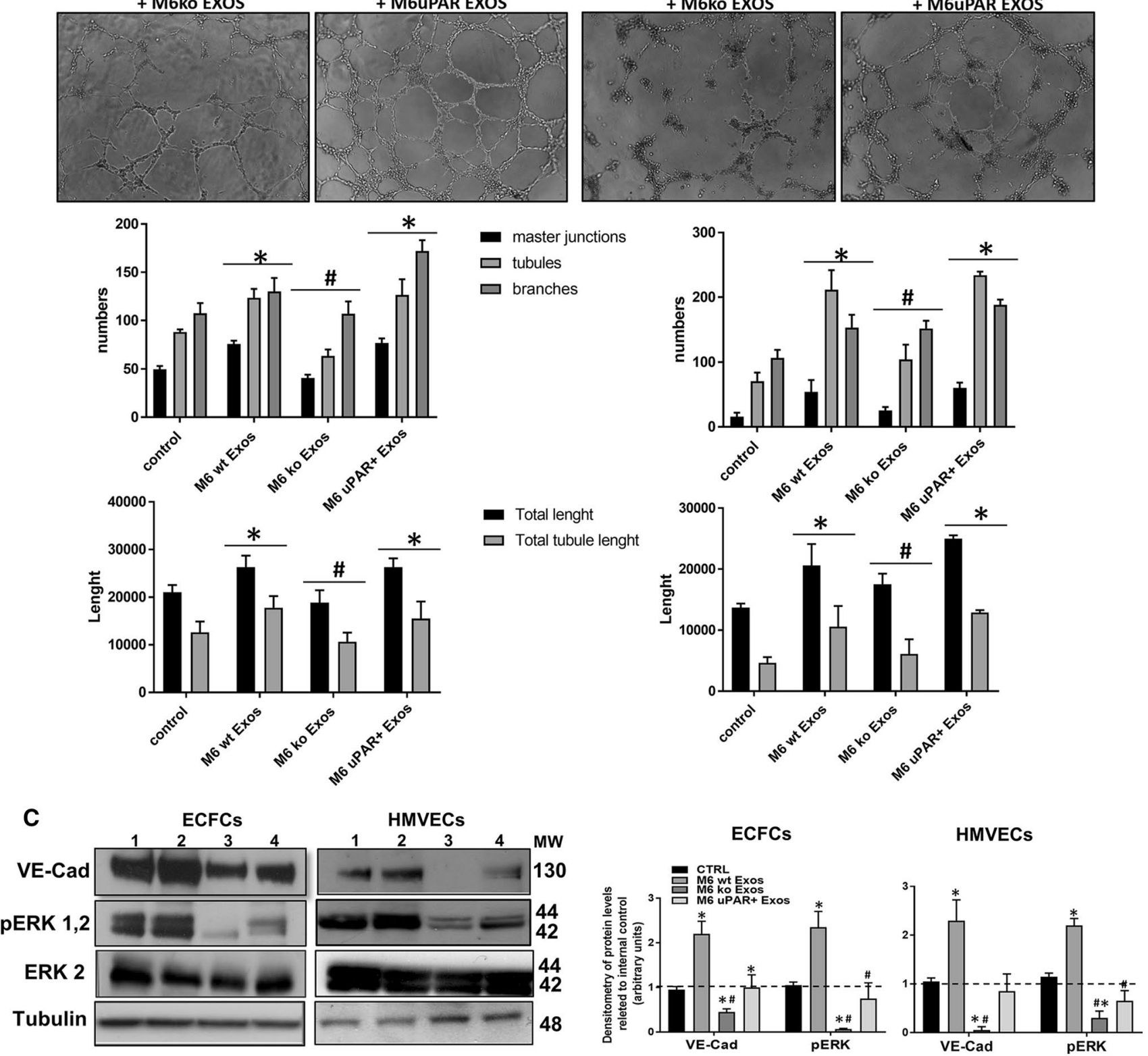

1= ctrl, 2= M6wt Exos, 3= M6ko Exos, 4= M6uPAR+ Exos 
4Fig. 7 Effects of uPAR knockout on the pro-angiogenic activities of melanoma Exos. a Western Blotting analysis of uPAR in M6-ko and M6-wt Exos (on the left) and in M6-ko and M6 u PAR+Exos (on the right). CD81 was used as loading control for exosomal samples. b Capillary morphogenesis assays in the presence of M6 wild-type Exos (M6-wt Exos), M6 uPAR Ko Exos (M6 Ko Exos) and uPAR rescued-Exos (M6 uPAR+Exos). Upper part: representative microphotographs $(\times 10)$ of capillary-like structures are shown. Lower part: quantification of capillary network by Angiogenesis Analyzer Image $\mathrm{J}$ tool. Histograms represent the mean number of master junctions, branches, tubules, total length, and total tubule length, respectively. Data are representative of measures obtained from at least nine fields. * Shows statistical significance $(p<0.05)$ compared to untreated cells. \# shows statistical significance $(p<0.05)$ compared to M6-wt Exos. c Western blotting analyses of VE-Cad, p ERK1,2, ERK1,2 and Tubulin in ECFCs and HMVECs in control conditions (ctrl) and treated with wild-type Exos (M6-wt Exos), M6 uPAR Ko Exos (M6 Ko Exos) and uPAR rescued-Exos (M6 uPAR+Exos). Densitometric quantification of the immunoblots normalized to the relative internal control and expressed respect to untreated (- M6 and A375 exos) ECFCs and HMVECs is reported on the right. $M W$ molecular weight

migration, invasion and capillary-like structure formation both in HMVECs and ECFCs. Considering the effect of A375 and M6-Exos in vivo, we observed that Exos from both parental cell lines promote endothelial cells recruitment and vascular organization within Matrigel plugs.

The uPA/uPAR system is essential for the endothelial function and for a correct angiogenic program. Indeed, we have previously reported that full-length UPAR is required for the angiogenic response of ECFCs [24]. At the same time, we have also shown that the MMP12-dependent uPAR cleavage is responsible for an angiogenesis impairment in HMVECs [42]. Here, to study the uPAR function in Exos, we exploited the CRISPR-Cas 9 technology to obtain a complete uPAR knockout, as recently published [29]. In this study, we reported that the UPAR silencing and, even more, the CRISPR-mediated knockout abrogate the pro-angiogenic potential of melanoma Exos both in vitro and in vivo. On the other hand, the pro-angiogenic ability of melanoma Exos is recovered by uPAR rescue, demonstrating a direct correlation between exosomal expression of UPAR and the proangiogenic properties of Exos.

During angiogenesis, adhesion molecules are important to provide connections between endothelial cells and to maintain the integrity of vascular tubes [43, 44]. The Vascular Endothelial Cadherin (VE-Cadherin) is a key adhesion molecule actively involved in the formation and stabilization of intercellular adherens junctions in endothelial cells and in modulating signaling cascades within endothelial cells during angiogenesis, vessel morphogenesis and vascular development [44]. Accordingly, also VEGF/
VEGFR2 interaction activates VE-Cadherin expression and the signaling pathway ERK/MAPK [45]. Here we have shown that the A375- and M6-Exos treatment induced an increase of VE-Cadherin, UPAR and EGFR protein levels both in mature and progenitor endothelial cells, in parallel with an increment of ERK1,2 phosphorylation, a signaling pathway that is inhibited by the peptide M25 which uncouples integrins-dependent UPAR interactions with receptor tyrosine kinases (RTKs), including EGFR [17, 18]. These data are in agreement with the paper of LaRusch GA et al. in which a $\beta 1$-integrin peptide that binds uPAR blocks FXII-induced angiogenesis inhibiting the ERK1,2 and Akt phosphorylation in human umbilical vein endothelial cells (HUVEC) [46].

In the presence of Exos from uPAR knockout parental cell lines, we observed a reduction of VE-Cadherin, and UPAR and EGFR expression, in parallel with a decrease of ERK1,2 phosphorylation. Again, VE-Cadherin, uPAR and EGFR levels and ERK1,2 signaling were restored in endothelial cells after UPAR rescue. Our data on Exos-dependent endothelial cell expression of EGFR, its phosphorylation and Gefitinibdependent inhibition of Exos-induced capillary morphogenesis (Fig. 6) are supported by several evidences that stimulation or inhibition of EGFR has significant consequences on tumor angiogenesis, a feature that involves both a direct effect and an interplay with VEGF [47-51]. In light of our results, it could be speculated that the reported abundance of EGFR (Erb-B1) on endothelial cells of tumor vessels [49, 50] could depend on an enrichment mediated by the release of Exos by the malignant cells.

Moreover, the crosstalk between uPAR and VE-Cadherin has been previously shown. Brunner et al. observed that in initial phases of angiogenesis, uPAR undergoes downregulation by density-enhanced phosphatase-1 (DEP-1) in confluent endothelial cells [52]. The up-regulation of DEP-1, with increasing cell density, inhibits the ERK1,2 pathway and UPAR expression in confluent endothelial cells, through VE-Cadherin/B-catenin interaction [53].

Collectively, our findings demonstrate that UPAR is critical for the Exos-mediated angiogenic program in human malignant melanoma and that the evaluation of exosomal uPAR expression and the complete uPAR knockout by gene editing technique may be a potential approach for monitoring and treatment of human melanoma. As a future perspective, this study provides new insights for a possible use of UPAR as a helpful biomarker in exosomal preparations obtained from the liquid biopsy in metastatic melanoma patients. 

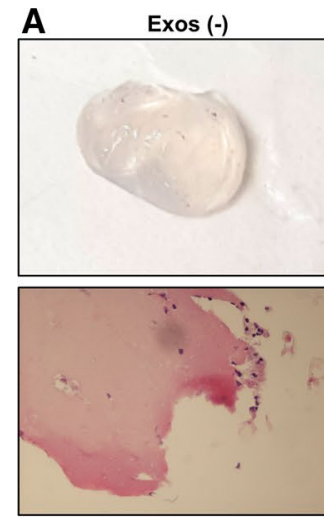

Exos (-)
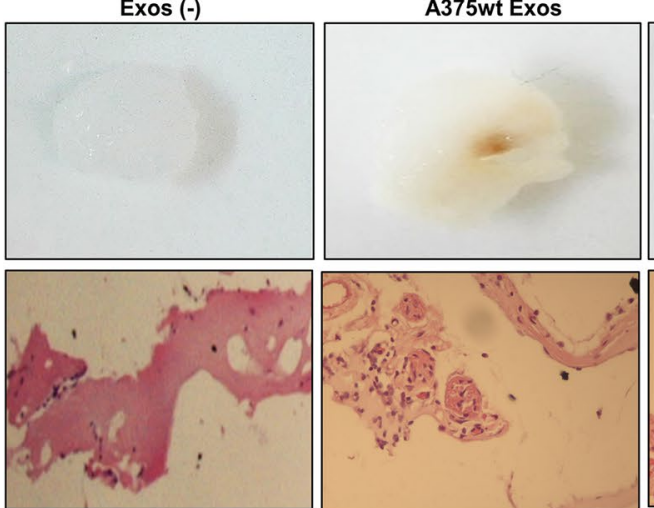

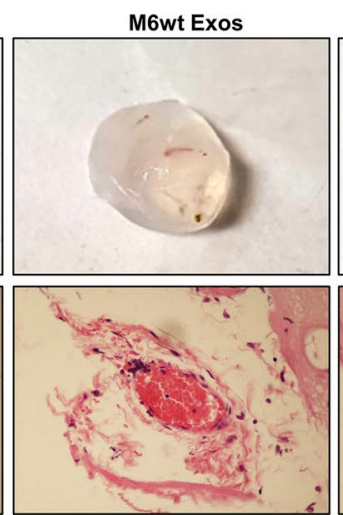

A375wt Exos

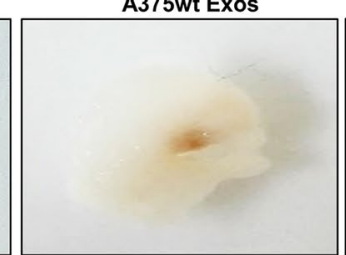

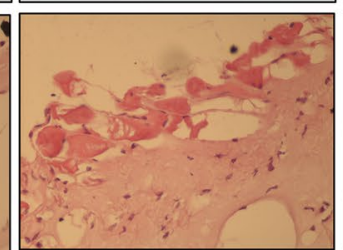

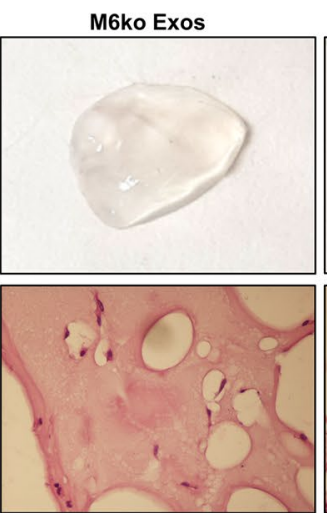

A375ko Exos
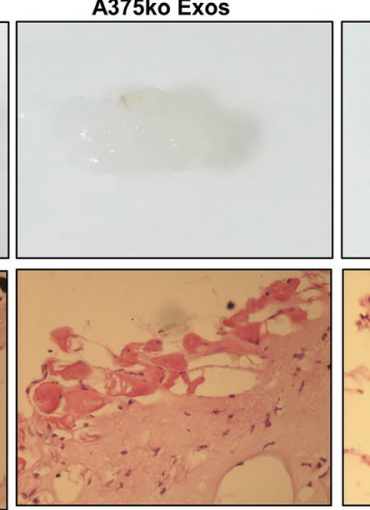

M6 UPAR+ Exos

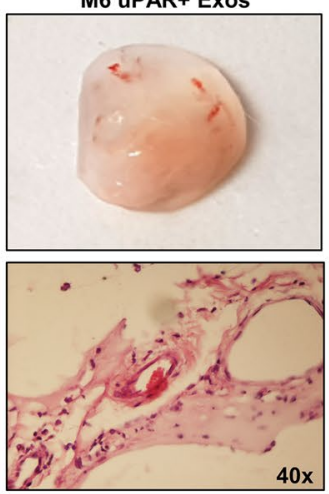

A375 UPAR+ Exos
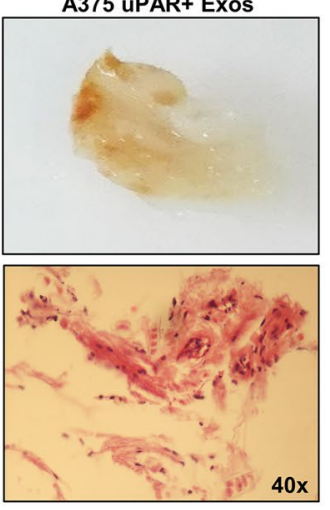

B
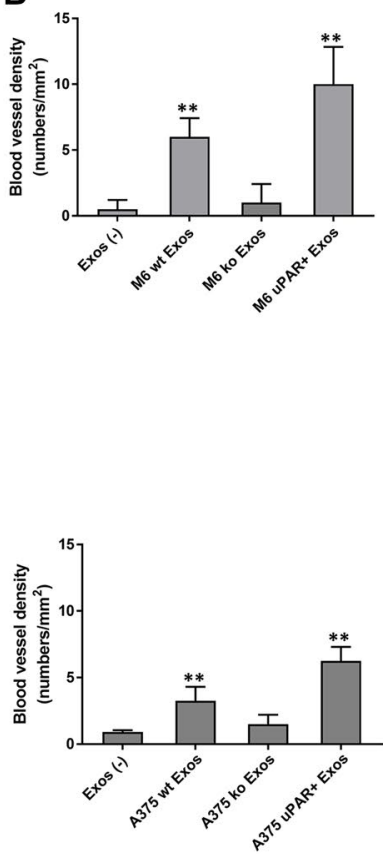

Fig. 8 uPAR+melanoma exosomes promote angiogenesis in vivo. Matrigel plug assay: Matrigel containing wt, ko and uPAR+Exos, or not, were injected subcutaneously in the nude mice. Representative images of the Matrigel plugs were shown (Fig. 7a). In vivo neovas-

Acknowledgements We would like to thank Prof. Daniele Bani of the Department of Experimental and Clinical Medicine-for TEM analysis.

Author contributions Conception and design of the study: F.M., A.C., G.F., M.D.R. Performing experiments: A.C., A.B., A.L., B.M. Methodology: F.M. Acquisition, analysis and interpretation: S.P., S.S., E.A., A.M., L.C., A.C. TRPS and Nanosight analyses: P.P. TEM analysis: D.G. Performing in vivo experiments F.B. and A.C. Critical review and drafting article: A.C., F.M. All authors read and approved the final manuscript.

Funding Open access funding provided by Università degli Studi di Firenze within the CRUI-CARE Agreement. This work was supported by Ente Cassa di Risparmio di Firenze (2016.1225) and Associazione Italiana per la Ricerca sul Cancro (IG 2013N. 14266). Dr. Alessio Biagioni and Dr. Elena Andreucci were supported by a post-doctoral fellowship of the Fondazione Italiana per la Ricerca sul Cancro (FIRC). Dr. Anastasia Chillà is a recipient of a Global Marie Curie Fellowship (October 2017-September 2020).

Data availability All the data generated or analyzed during this study are included in this published article and its supplementary files. The datasets and materials in the current study available from the corresponding author on reasonable request. cularization induced by M6- and A375-Exos was evaluated by Hematoxylin and Eosin staining and quantified by blood vessel density (Fig. 7b) using Image $\mathrm{J}$ software

\section{Compliance with ethical standards}

Conflict of interest The authors declare no competing interest.

Ethical approval Animal experiments, transportation, and care were conducted in compliance with the relevant laws and the guidelines issued by the Ethical Committee of University of Florence.

Consent to participate All authors of this article have directly participated in the planning and drafting and all authors listed have read and approved the final version including details and images.

Consent for publication The written informed consent for the publication has been obtained from all the authors.

Open Access This article is licensed under a Creative Commons Attribution 4.0 International License, which permits use, sharing, adaptation, distribution and reproduction in any medium or format, as long as you give appropriate credit to the original author(s) and the source, provide a link to the Creative Commons licence, and indicate if changes were made. The images or other third party material in this article are included in the article's Creative Commons licence, unless indicated otherwise in a credit line to the material. If material is not included in the article's Creative Commons licence and your intended use is not permitted by statutory regulation or exceeds the permitted use, you will need to obtain permission directly from the copyright holder. To view a copy of this licence, visit http://creativecommons.org/licenses/by/4.0/. 


\section{References}

1. Fleming NH, Zhong J, Da Silva IP, Vega-Saenz E, de Miera B, Brady SWH, Hanniford D, Wang J, Shapiro RL, Hernando E, Osman I (2015) Serum-based miRNAs in the prediction and detection of recurrence in melanoma patients. Cancer 121:5159. https://doi.org/10.1002/cncr.28981

2. Prado G, Svoboda RM, Rigel DS (2019) What's new in melanoma. Dermatol Clin 37(2):159-168. https://doi.org/10.1016/j. det.2018.12.005

3. Leonardi GC, Falzone L, Salemi R, Zanghi A, Spandidos DA, McCubrey JA, Candido S, Libra MM (2018) Cutaneous melanoma: from pathogenesis to therapy. Int J Oncol 52(4):1071-1080. https://doi.org/10.3892/ijo.2018.4287

4. Fidler IJ (2015) The biology of brain metastasis: challenges for therapy. Cancer J 21(4):284-293. https://doi.org/10.1097/ PPO.0000000000000126

5. Isola AL, Eddy K, Chen S (2016) Biology, therapy and implications of tumor exosomes in the progression of melanoma. Cancers 8(12):110. https://doi.org/10.3390/cancers 8120110

6. Tucci M, Mannavola F, Passarelli A, Stucci LS, Cives M, Silvestris F (2018) Exosomes in melanoma: a role in tumor progression, metastasis and impaired immune system activity. Oncotarget 9:20826-20837. https://doi.org/10.18632/oncotarget.24846

7. Ciardiello C, Cavallini L, Spinelli C, Yang J, Reis-Sobreiro M, De Candia P, Minciacchi VR, Di Vizio D (2016) Focus on extracellular vesicles: new frontiers of cell-to-cell communication in cancer. Int J Mol Sci 6:175. https://doi.org/10.3390/ijms170201 75

8. Greening DW, Xu R, Gopal SK, Rai A, Simpson RJ (2016) Proteomic insights into extracellular vesicle biology-defining exosomes and shed microvesicles. Expert Rev Proteom 14:69-95. https://doi.org/10.1080/14789450.2017.1260450

9. Zhang H, Freitas D, Kim HS, Fabijanic K, Li Z, Chen H et al (2018) Identification of distinct nanoparticles and subsets of extracellular vesicles by asymmetric flow field-flow fractionation. Nat Cell Biol 20:332-343. https://doi.org/10.1038/s41556-018-0040-4

10. Hood JL (2019) Natural melanoma-derived extracellular vesicles. Semin Cancer Biol 59:251-265. https://doi.org/10.1016/j.semca ncer.2019.06.020

11. Record M, Carayon K, Poirot M, Silvente-Poirot S (2013) Exosomes as a vesicular transporters involved in cell-cell communication and various pathophysiologies. Biochim Biophys Acta 1841:108-120. https://doi.org/10.1016/j.bbalip.2013.10.004

12. Simons M, Raposo G (2009) Exosomes-Vesicular carriers for intercellular communication. Curr Opin Cell Biol 21(21):575581. https://doi.org/10.1016/j.ceb.2009.03.007

13. Mannavola F, D’Oronzo S, Cives M, Stucci LS, Ranieri G, Silvestris F, Tucci M (2019) Extracellular vesicles and epigenetic modifications are hallmarks of melanoma progression. Int J Mol Sci 20:52. https://doi.org/10.3390/ijms21010052

14. Schmitt M, Mengele K, Napieralski R, Magdolen V, Reuning U, Gkazepis A, Sweep F, Brünner N, Foekens J, Harbeck N (2010) Clinical utility of level-of-evidence-1 disease forecast cancer biomarkers uPA and its inhibitor PAI-1. Expert Rev Mol Diagn 10(2010):1051-1067. https://doi.org/10.1586/erm.10.71

15. Ulisse S, Baldini E, Sorrenti S, D’Armiento M (2009) The urokinase plasminogen activator system: a target for anti-cancer therapy. Curr Cancer Drug Targets 9:32-71

16. Laurenzana A, Biagioni A, Bianchini F, Peppicelli S, Chillà A, Margheri F, Luciani C, Pimpinelli N, Del Rosso M, Calorini L, Fibbi G (2015) Inhibition of uPAR-TGF $\beta$ crosstalk blocks MSCdependent EMT in melanoma cells. J Mol Med 93:783-794. https ://doi.org/10.1007/s00109-015-1266-2
17. Laurenzana A, Chillà A, Luciani C, Peppicelli S, Biagioni A, Bianchini F, Tenedini E, Torre E, Mocali A, Calorini L, Margheri F, Fibbi G, Del Rosso M (2017) uPA/uPAR system activation drives a glycolytic phenotype in melanoma cells. Int J Cancer 141:1190-1200. https://doi.org/10.1002/ijc.30817

18. Laurenzana A, Margheri F, Biagioni A, Chillà A, Pimpinelli N, Ruzzolini J, Peppicelli S, Andreucci E, Calorini L, Serratì S, Del Rosso M, Fibbi G (2019) EGFR/uPAR interaction as druggable target to overcome vemurafenib acquired resistance in melanoma cells. EBioMedicine 39:194-206. https://doi.org/10.1016/j.ebiom .2018.12.024

19. Cobos E, Jumper C, Lox C (2003) Pretreatment determination of the serum urokinase plasminogen activator and its soluble receptor in advanced small-cell lung cancer or non-small-cell lung cancer. Clin Appl Thromb Hemost 9:241-246

20. Riisbro R, Christensen IJ, Piironen T, Greenall M, Larsen B, Stephens RW, Han C, Høyer-Hansen G, Smith K, Brünner N, Harris AL (2002) Prognostic significance of soluble urokinase plasminogen activator receptor in serum and cytosol of tumor tissue from patients with primary breast cancer. Clin Cancer Res 8:1132-1141

21. Liu KL, Luo JL, Wu J, Wang YD, Fan HJ (2017) A Preliminary Study of the Value of Plasma microRNA-193b and Soluble Urokinase-Type Plasminogen Activator Receptor in Identifying Patients with Early-Stage Colorectal Cancer. Clin Lab 63:1949-1953. https ://doi.org/10.7754/Clin.Lab.2017.170726

22. Leandersson P, Kalapotharakos G, Henic E, Borgfeldt H, Petzold M, Høyer-Hansen G, Borgfeldt C (2016) A biomarker panel increases the diagnostic performance for epithelial ovarian cancer Type I and II in young women. Anticancer Res 36:957-965

23. Wach S, Al-Janabi O, Weigelt K, Fischer K, Greither T, Marcou M, Theil G, Nolte E, Holzhausen HJ, Stöhr R, Huppert V, Hartmann A, Fornara P, Wullich B, Taubert H (2015) The combined serum levels of miR-375 and urokinase plasminogen activator receptor are suggested as diagnostic and prognostic biomarkers in prostate cancer. Int J Cancer 15:1406-1416. https://doi. org/10.1002/ijc. 29505

24. Margheri F, Chillà A, Laurenzana A, Serratì S, Mazzanti B, Saccardi R, Santosuosso M, Danza G, Sturli N, Rosati F, Magnelli L, Papucci L, Calorini L, Bianchini F, Del Rosso M, Fibbi G (2011) Endothelial progenitor cell-dependent angiogenesis requires localization of the full-length form of UPAR in caveolae. Blood 118:3743-3755. https://doi.org/10.1182/blood-2011-02-338681

25. Hong CS, Funk S, Muller L, Boyiadzis M, Whiteside TL (2016) Isolation of biologically active and morphologically intact exosomes from plasma of patients with cancer. J Extracell Vesicles 5:29289. https://doi.org/10.3402/jev.v5.29289

26. Shu S, Yang Y, Allen CL, Maguire O, Minderman H, Sen A, Ciesielski MJ, Collins KA, Bush PJ, Singh P, Wang X, Morgan M, Qu J, Bankert RB, Whiteside TL, Wu Y, Ernstoff MS (2018) Metabolic reprogramming of stromal fibroblasts by melanoma exosome microRNA favours a pre-metastatic microenvironment. Sci Rep 8:12905. https://doi.org/10.1038/s41598-018-31323-7

27. Mutschelknaus L, Peters C, Winkler K, Yentrapalli R, Heider T, John Atkinson M, Moertl S (2016) Exosomes derived from squamous head and neck cancer promote cell survival after ionizing radiation. PLoS ONE 11(3):E0152213. https://doi.org/10.1371/ journal.pone. 0152213

28. Margheri F, Manetti M, Serrati S, Nosi D, Pucci M, MatucciCerinic M, Kahaleh B, Bazzichi L, Fibbi G, Ibba-Manneschi L, Del Rosso M (2006) Domain 1 of the urokinase-type plasminogen activator receptor is required for its morphologic and functional, beta2 integrin-mediated connection with actin cytoskeleton in human microvascular endothelial cells: failure of association in systemic sclerosis endothelial cells. Arthritis Rheum 54:3926-3938 
29. Biagioni A, Laurenzana A, Chillà A, Del Rosso M, Andreucci E, Poteti M, Bani D, Guasti D, Fibbi G, Margheri F (2020) uPAR knockout results in a deep glycolytic and OXPHOS reprogramming in melanoma and colon carcinoma. Cells 28:E308. https://doi.org/10.3390/cells9020308

30. Pucci M, Fibbi G, Magnelli L, Del Rosso M (2001) Regulation of urokinase/urokinase receptor interaction 421 by heparin-like glycosaminoglycans. J Biol Chem 276:4756-4765

31. Surman M, Kedracka-Krok S, Hoja-Łukowicz D, Jankowska U, Drozdz A, E Ł. Stepie'n, M Przybyło, (2020) Mass spectrometry-based proteomic characterization of cutaneous melanoma ectosomes reveals the presence of cancer-related molecules. Int J Mol Sci 21(8):2934. https://doi.org/10.3390/ijms21082934

32. Ekstrom EJ, Bergenfelz C, Von Bulow V, Serifler F, Carlemalm E, Jönsson G, Andersson T, Leandersson K (2014) WNT5A induces release of exosomes containing pro-angiogenic and immunosuppressive factors from malignant melanoma cells. Mol Cancer 13:88. https://doi.org/10.1186/1476-4598-13-88

33. Peinado H, Alec `ković M, Lavotshkin S, Matei I, Costa-Silva B, Moreno-Bueno G, Hergueta-Redondo M, Williams C, GarcíaSantos G, Ghajar C, Nitadori-Hoshino A, Hoffman C, Badal K, Garcia BA, Callahan MK, Yuan J, Martins VR, Skog J, Kaplan RN, Brady MS, Wolchok JD, Chapman PB, Kang Y, Bromberg J, Lyden D (2016) Melanoma exosomes educate bone marrow progenitor cells toward a pro-metastatic phenotype through MET. Nat Med 22:1502. https://doi.org/10.1038/nm.2753

34. Margheri F, Papucci L, Schiavone N, D’Agostino R, Trigari S, Serratì S, Laurenzana A, Biagioni A, Luciani C, Chillà A, Andreucci E, Del Rosso T, Margheri G, Del Rosso M, Fibbi G (2015) Differential uPAR recruitment in caveolar-lipid rafts by GM1 and GM3 gangliosides regulates endothelial progenitor cells angiogenesis. J Cell Mol Med 19:113-123. https://doi. org/10.1111/jcmm. 12410

35. Thuma F, Heiler S, Schnölzer M, Zöller M (2014) Palmitoylated claudin 7 captured in glycolipid-enriched membrane microdomains promotes metastasis via associated transmembrane and cytosolic molecules. Oncotarget 21:30659-30677. https://doi. org/10.18632/oncotarget.8928

36. Lajoie P, Nabi IR (2010) Lipid rafts, caveolae, and their endocytosis. Int Rev Cell Mol Biol 282:135-163. https://doi.org/10.1016/ S1937-6448(10)82003-9

37. Ewers H, Helenius A (2011) Lipid-mediated endocytosis. Cold Spring Harb Perspect Biol 3:a004721. https://doi.org/10.1101/ cshperspect.a004721

38. Zöller M (2009) Tetraspanins: push and pull in suppressing and promoting metastasis. Nat Rev Cancer 9:40-55. https://doi. org/10.1038/nrc2543

39. Nicolson GL (2015) Cell membrane fluid-mosaic structure and cancer metastasis. Cancer Res 75:1169-1176. https://doi. org/10.1158/0008-5472.CAN-14-3216

40. ZAndreu, M Yáñez-Mó, (2014) Tetraspanins in extracellular vesicle formation and function. Front Immunol 5:442. https:// doi.org/10.3389/fimmu.2014.00442

41. Endo-Munoz L, Cai N, Cumming A, Macklin R, Merida L, de Long E, Topkas PM, Hill M, Saunders SA (2015) Progression of osteosarcoma from a non-metastatic to a metastatic phenotype is causally associated with activation of an autocrine and paracrine
uPA axis. PLoS ONE 10:e0133592. https://doi.org/10.1371/journ al.pone. 0133592

42. D’Alessio S, Fibbi G, Cinelli M, Guiducci S, Del Rosso A, Margheri F, Serratì S, Pucci M, Kahaleh B, Fan P, Annunziato F, Cosmi L, Liotta F, Matucci-Cerinic M, Del Rosso M (2004) Matrix metalloproteinase 12-dependent cleavage of urokinase receptor in systemic sclerosis microvascular endothelial cells results in impaired angiogenesis. Arthritis Rheum 50:3275-3285

43. Bentley K, Franco CA, Philippides A, Blanco R, Dierkes M, Gebala V, Stanchi F, Jones M, Aspalter IM, Cagna G, Weström S, Claesson-Welsh L, Vestweber D, Gerhardt H (2014) The role of differential VE-cadherin dynamics in cell rearrangement during angiogenesis. Nat Cell Biol 16:309-321. https://doi.org/10.1038/ ncb2926

44. Lagendijk AK, Hogan BM (2015) Chapter ten VE-cadherin in vascular development: a coordinator of cell signaling and tissue morphogenesis. Curr Top Dev Biol 112:325-352. https://doi. org/10.1016/bs.ctdb.2014.11.024

45. Yamaguchi K, Sudo H, Imai K (2019) Vascular endothelial growth factor signaling in VE-cadherin expression and tubelike formation by rheumatoid arthritic synovial fibroblast-like cells. Biochem Biophys Res Commun 508:405-409. https://doi. org/10.1016/j.bbrc.2018.11.116

46. LaRusch GA, Mahdi F, Shariat-Madar Z, Adams G, Sitrin RG, Zhang WM, McCrae KR, Schmaier AH (2010) Factor XII stimulates ERK1/2 and Akt through uPAR, integrins, and the EGFR to initiate angiogenesis. Blood 115(24):5111-5120. https://doi. org/10.1182/blood-2009-08-236430

47. Ellis LM (2004) Epidermal growth factor receptor in tumour angiogenesis. Hematol Oncol Clin N Am 18:1007-1021. https:// doi.org/10.1016/j.hoc.2004.06.002

48. Van Cruijsen G, Hoekm K (2006) Epidermal growth factor receptor and angiogenesis: opportunities for combined anticancer strategies. Int J Cancer 118:883-888

49. Hirata A, Uehara H, Izumi K, Naito S, Kuwano M, Ono M (2004) Direct inhibition of EGF receptor activation in vascular endothelial cells by gefitinib ('Iressa', ZD1839). Cancer Sci 95:614-618

50. Amin DN, Hida K, Bielenberg DR, Klagsbrun M (2006) Klagsbrun (2006) Tumor endothelial cells express epidermal growth factor receptor (EGFR) but not ErbB3 and are responsive to EGF and to EGFR kinase inhibitors. Cancer Res 66:2173-2180

51. Maretzky T, Evers A, Zhou W, Swendeman SL, Wong P-M, Rafii S, Reiss K, Blobel CP (2011) Migration of growth factor-stimulated epithelial and endothelial cells depends on EGFR transactivation by ADAM17. Nat Commun 2:229-254

52. Brunner PM, Heier PC, Mihaly-Bison J, Priglinger U, Prager GW, Binder BR (2011) Density enhanced phosphatase-1 (DEP1) down-regulates urokinase receptor (UPAR) surface expression in confluent endothelial cells. Blood 117:4154-4161. https://doi. org/10.1182/blood-2010-09-307694

53. Del Rosso M (2011) uPAR in angiogenesis regulation. Blood 117:3941-3943. https://doi.org/10.1182/blood-2011-02-337733

Publisher's Note Springer Nature remains neutral with regard to jurisdictional claims in published maps and institutional affiliations. 